\title{
Optimal Power Control, Scheduling and Routing in UWB Networks
}

\author{
Božidar Radunović, Student Member, IEEE, and Jean-Yves Le Boudec, Fellow, IEEE,
}

\begin{abstract}
Ultra-Wide Band (UWB) is an emerging wireless physical layer technology that uses a very large bandwidth. We are interested in finding the design objectives of the medium access (MAC, namely, power control and scheduling) and routing protocols of a multi-hop, best-effort, UWB network. Our objective is to maximize flow rates (more precisely, log-utility of flow rates) given node power constraints. The specificity of UWB is expressed by the linear dependence between rate and signal-to-noise ratio at the receiver. It is known that, in wireless networks, different routing strategies can imply differences in MAC protocol design. Hence we search for the jointly optimal routing, scheduling and power control.

We find that the optimal solution is characterized by the following. (1) When data is being sent over a link, it is optimal to have an exclusion region around the destination, in which all nodes remain silent during transmission, whereas nodes outside of this region can transmit in parallel, regardless of the interference they produce at the destination. Additionally, the source adapts its transmission rate according to the level of interference at the destination due to sources outside of the exclusion region. (2) The optimal size of this exclusion region depends only on the transmission power of the source of the link, and not on the length of the link nor on positions of nodes in its vicinity. (3) Each node in a given time slot either sends data at the maximum power, or does not send at all. As for the routing, we restrict ourselves to a subset of routes where on each successive hop we decrease the distance toward the destination, and we show that (4) relaying along a minimum energy and loss route is always better than using longer hops or sending directly, which is not obvious since we optimize rate and not power consumption. Finally (5), the design of the optimal MAC protocol is independent of the choice of the routing protocol. For narrow-band networks, (2), (4) and (5) do not hold, which shows that the design of an UWB network should be addressed in a different way than for narrow-band.

Our technical approach is based on expressing the design requirements as a mathematical optimization problem. We solve it exactly for simple networks on a line and approximately on random topologies in a plane with up to 50 nodes with various power constraints, traffic matrices, and mobility parameters.
\end{abstract}

\section{INTRODUCTION}

\section{A. Goals}

Our goal is to define design objectives for multi-hop ultrawide band (UWB) best effort ad-hoc networks. We want to understand how access to the radio medium should be organized (medium access control or MAC protocol), how to choose the optimal routing, and how the two problems interact. Indeed, as we explain in the next section, UWB has

Manuscript received October 1, 2003; revised March 15, 2004. The work presented in this paper was supported (in part) by the National Competence Center in Research on Mobile Information and Communication Systems (NCCR-MICS), a center supported by the Swiss National Science Foundation under grant number 5005-67322. Authors are with EPFL, School of Computer and Communication Sciences, 1015 Ecublenes, Switzerland (e-mail: bozidar.radunovic@epfl.ch; jean-yves.leboudec@epfl.ch). peculiarities that suggest that the optimal organization might be different than for existing, narrow-band wireless networks, and this is confirmed by our findings. We are interested in formulating fundamental tradeoffs, and we expect our results to serve as guidelines for protocol design. The application of our findings to the design of a specific MAC or routing protocol is outside the scope of this paper.

\section{B. Physical Layer Properties}

Ultra-wide band (UWB) is an emerging radio technology for wireless networks. According to the FCC, an ultra-wide band transmission has a bandwidth that is larger than $25 \%$ of the carrier frequency. There are currently several proposals of the physical layer for UWB, networks such as those based on coherent receivers [33], [1] and non-coherent receivers [25].

Despite a large diversity in the existing proposals, they all have in common [31], [28] that the Shannon capacity of the optimal wide-band radio is a linear function of the SNR at the receiver. From the implementation point of view, linear rate function means that given a fixed desired error probability, the maximal achievable rate is a linear function of SNR at the receiver. This property holds for all the existing proposals (e.g. [33], [1], [25]).

We also assume that the physical layer allows for rate adaptation: a sender uses variable coding to adapt the data rate to the channel conditions. One can use repetition coding, as in [33], or more advanced convolutional codes [12], [8], [1]. By increasing the power of the code, the sender increases the effective SNR at the receiver, hence decreasing the probability of error, but at the same time decreasing the rate, and vice versa. The rate can be very fine-tuned since this is done at the symbol level.

A consequence of rate adaptation is that an arbitrary level of interference is possible. For a given level of interference at the receiver, a sender can tune its rate by adjusting the code, in order to achieve a desired error probability. This way, a sender can avoid collisions while still obtaining the maximum possible rate for a given interference level. However, as we show later in the paper, allowing for an arbitrary level of interference may still not be optimal.

Our model holds for all multi-hop wireless networks whose underlying physical layers satisfy the three conditions from above. This includes [33], [1], as explained in Section III, and potentially other UWB implementations. In cases where rate can be adapted only coarsely to the signal-to-noise ratio, our solution is only approximately optimal. The UWB model of [25] differs from ours in a number of features. For more discussion see Section IX. 
An 802.11 network does not allow for an arbitrary level of interference and adaptive coding, and we show that the MAC protocol design paradigm in our case is significantly different than the 802.11 one [11].

Although the adaptive coding techniques can be applied to a narrow-band system, the linear rate function is a particularity of wide-band systems only. The model therefore does not hold for narrow-band systems (like 802.11 or CDMA). For more discussion see Section VIII.

\section{Cross-Layer Design in Wireless Networks}

Access to the radio medium is traditionally considered a problem of the MAC layer. However, it has recently become evident that a traditional layering approach that separates routing, scheduling, fbw and power control might not be efficient for ad-hoc wireless networks [10]. This is primarily due to the interaction of links through interference, which implies that a change in power allocation or schedules on one link can induce changes in capacities of all links in the surrounding area and changes in the performance of fbws that do not use the modified link. One example is given in [22], for a one-dimensional narrow-band network; it is shown that an ideal MAC protocol should establish an exclusion region around a destination of a transmitting link. The size of the region is proportional to the length of the link, hence the exclusion regions are going to be larger if nodes use direct routes instead of next-hop routes. Another example of interaction between the optimal routing and the optimal MAC protocol is given in [30] in the case of CSMA/CA networks. We are interested in finding a jointly optimal routing and MAC strategies, and we formulate our question as that of a joint optimization of power control, scheduling and routing.

\section{Performance Metric}

Performance metrics for wireless networks can be divided in two groups: rate based and power based metrics. Here we are primarily interested in maximizing rates and we consider power control a mechanism for increasing a rate-based performance metric, rather than saving battery lifetime (though it may come as a desirable offshoot). The most widely used rate-based performance objectives are total throughput, total utility, and max-min fairness.

The UWB physical layer has the property that the rate is a strictly increasing function of signal-to-noise level at the receiver. It is shown in [23] that for this type of network both total throughput and max-min fairness are not appropriate. The former suffers from unfairness and the latter from inefficiency. As suggested in [23], a good compromise between efficiency and fairness is achieved by proportional fairness [17]. Thus we use proportional fairness as a performance metric to define an optimal network design.

Although in this paper we focus on rate-maximization performance metrics, we note that the power consumption is also an important performance metric for wireless ad-hoc networks. Not considering powers in a performance metric may lead to battery drain of some highly utilized nodes. However, there is a whole direction in research that studies incentives for cooperations in wireless networks. We assume here that some of these schemes are implemented in the network, hence when a node is not willing to cooperate for various reasons (such as extensive battery drain) will deny it services and will not be visible any more for nodes asking the service.

\section{E. Routing and Mobility}

The optimal route of a fbw in the context defined above maximizes the utility of the system, and at the same time attains the Wardrop equilibrium [17]. This route is not necessarily close to the shortest route as it depends on rates and routes of other fbws.

In order to simplify the problem, conventional routing protocols like AODV [21] and DSR [14] focus on a set of routes that have as a next hop a node closer to the destination. We adopt this approach. We focus on routes that consist of nodes that lay on a "minimum energy and loss route" (MELR). The MELR of a fbw is defined as the route that maximizes the rate of the fbw in a hypothetical network with a single fbw where only one node can send at a time; for a more precise definition of MELR see Section IV-C. We optimize routing by tuning the length of the hop. One extreme example of such a route is the MELR route itself, when the smallest possible hop lengths are used; another extreme is the direct route where a source does not relay but sends directly to the destination.

Any implementation of routing incurs an additional penalty on network performance. There is overhead due to the implementation of the protocol. In order to maintain routes, a routing protocol needs to exchange beacons and keep-alive messages, thus consuming a part of network resources. We assume this overhead is fixed for each hop and we express it through a fraction of the rate it consumes.

Another penalty imposed by routing is due to the mobility of nodes. The longer the path is, the higher the probability that the path will break is, since one or more nodes will move. This probability depends, to a large extent, on the mobility pattern and speed, as well as on the properties of the implementation of the UWB physical layer on senders and receivers. We avoid in-depth modeling of these factors because the goal of this paper is to anticipate to which extent mobility influences the optimal choice of routes in an UWB network. We incorporate the impact of mobility through the probability that a packet is lost at each hop of the route.

\section{F. Problem Formulation}

We define the scheduling, routing and power allocation problem in a UWB network as a numerical optimization problem, whose goal is to maximize the total utility of the system. The questions we pose are:

- How to choose routes for fbws ? - Given an arbitrary set of nodes and traffic demands, what is the routing that achieves proportional fairness? What is the optimal hop length of a fbw at each intermediate node?

- How to organize the access to the network (MAC protocol) ? - Given the optimal routing, when and with what rate and power should a link transmit? While a link is transmitting, how should it control its power, the 
interference at the receiver, and how should it adapt its rate?

- How do routing and MAC protocols interact ? - How does the optimal MAC protocol depend on the choice of the routing protocol and vice versa?

Due to high complexity, it is not possible to solve this problem exactly in a general case. We solve it using a combination of analytical and numerical techniques. This is explained in more details in Section IV-D.

\section{G. Our Findings}

Our main findings can be summarized in the following 5 items:

1) Exclusion Regions and Rate Adaptation: Although a link can adapt the rate to an arbitrary level of interference at the receiver, this might not be optimal from the rate viewpoint. It is optimal to have an exclusion region around the destination of an active link. All nodes in the exclusion region have to remain silent. However, nodes outside of the exclusion region can transmit data in parallel. The destination will thus experience interference only from nodes outside of the exclusion region, and will adapt the rate accordingly. This is very much in contrast to the 802.11 CSMA/CA mechanism, where an exclusion region is defined around the source and the destination of a link at the same time, and where the size of the exclusion region is adapted such that the interference at the receiver is smaller than a threshold, while the rate is kept fixed.

2) Static Size of The Exclusion Region: The size of this exclusion region around a destination is static. It depends only on the transmission power constraint of the corresponding source, and not on the link length nor on the density of nodes around the destination.

From this conclusion we derive two interesting extreme cases. For small power constraints, hence low-rate or very sparse networks, we can schedule as many nodes as possible to send at a time, even if it increases interference. This finding suggests that a simple MAC protocol should be used in this type of network, as only the sender and the receiver of each link need to coordinate separately. In contrast, for large power constraints and high-rate networks, or very dense networks, it is optimal to have only one node sending at a time.

3) Sending at The Full Power: When data is sent over a link, the sender should transmit with the maximum allowed power. Otherwise, the link should remain silent. This is because the rate on a UWB link can always increase when the SNR at the receiver is increased, even if this increase is small. Even though an increase of a transmission power will increase interference at other nodes sending at the same time, we find that this will always be compensated by the increase of the rate on the link itself.

This is again in contrast with 802.11 , where a source can use only one of a few modulations to send data. The source selects the modulation, and hence the rate, a priori based on the link conditions. It is then shown in [16] that a sender should send with the minimal power necessary to reach the SNR threshold of the receiver, in order to decrease the interference on others and maximize performance.
4) Minimum Energy and Loss Routing: The routing that maximizes total rate utility in a UWB network within the routes we consider is the minimum energy and loss route itself. In addition, if maximum power constraints are uniform, and nodes are not mobile, this optimal route coincides with what is usually called the minimum energy route (MER), described in Section IV-C. This result is similar to the findings in 802.11 networks, as shown in [16].

5) Insensitivity of MAC to The Choice of Routes: Another significant conclusion is that the optimal MAC protocol does not depend on the choice of routes. We found that if all nodes apply similar routing strategies, then the MAC protocol should maintain exclusion regions of the fixed sizes, regardless of the choice of hop lengths made by routing. This leads to an important property: a UWB network can be organized in a traditional layered manner, where a routing protocol choose routes, and a MAC protocol organizes medium access regardless of the choice of routes. This conclusion is in contradiction with findings for 802.11 [30] and narrow-band networks in general [22], and facilitates the design of a UWB network protocol.

The four main findings presented above are fundamentally related to the properties of the UWB physical layer presented in Section I-B. For a discussion on application of the findings on the UWB model of [25], see Section IX.

The findings do not hold for narrow-band physical layers like CDMA or 802.11, as we show Section VIII. Thus the fact that the rate is a linear function of the signal-to-noise ratio is important.

\section{H. Organization of This Paper}

In the following section we present existing work related to our research. In Section III we define our assumptions and describe our network model in details; we translate our initial problem into a numerical optimization problem. In Section V we solve the numerical problem exactly for a static linear network. In Section VI we present an approximate method for solving the general case of a mobile network in the plane and in Section VII we discuss the numerical results. Finally, in Section IX we give conclusions and directions for future work.

\section{RELATED WORK}

There are several papers that study variations of the joint optimization problem. In [7] the joint scheduling and power control problem is considered in networks with QoS constraints, where a minimum signal to noise ratio is defined for every link. Given these set of constraints, they find an optimal scheduling and power allocation that satisfies constraints and minimizes dissipated power. A similar model with a minimum SIR constraint is analyzed in [3].

In [5] the authors consider a UWB network without scheduling (with a single power allocation). They consider both QoS and best effort traffic. They show that in the optimal power allocation, which maximizes the total throughput of a network, each node sends with full power or does not send at all. 
In [4], the authors consider a network with scheduling and best-effort traffic. They search for the optimal scheduling and power control that maximizes the total throughput of a network, given the power constraints for each user. They show that the optimal power control consists of sending with maximum power. They further solve the scheduling problem for small networks and they demonstrate a distributed algorithm that finds an approximate solution for large networks based on hierarchy.

Joint routing and scheduling is considered in [26], where the total throughput of a network is maximized when given a set of links that cannot be active at the same time.

In [29] the authors define a very general model of a wireless network that covers both routing, scheduling and power control. They take the total network capacity as a performance measure. However, the complexity of the model is such that even with a linear objective function it can handle less than 10 nodes.

Asymptotic performance of UWB networks is discussed in [24]. The global organization of UWB networks is studied in [2] in a guaranteed service framework where fbws are setup using a centralized control system and where scheduling allows all nodes to send together. In this paper we focus on best-effort networks. In most of the papers concerning optimal performance of best-effort wireless networks for different physical layers, such as [7], [3], [4], [26], [29], the authors maximize total throughput of the network. This approach can lead to gross unfairness, where users with worse channels might not get any throughput. Per-link utility fairness has been considered in [20], and per-link max-min fairness in [27], [13]. However, this still does not guarantee per-fbw fairness for long fbws.

\section{Assumptions AND MODELING}

\section{A. Notations}

We model the wireless network as a set of $I$ fbws, $L$ links, $O$ nodes and $N$ time-slots. Flows are unicast or multicast. We give here a list of notations used in this section to describe the model. The precise definitions are given in subsequent subsections.

- $\mathbf{f} \in \mathbb{R}^{I}$ is the vector of average rates achieved by fbws.

- $\overline{\mathbf{x}} \in \mathbb{R}^{L}$ is the vector of average rates achieved on links.

- for every $n \in\{1, \cdots, N\}, \mathbf{x}^{n} \in \mathbb{R}^{L}$ is the vector of rates achieved on links in time slot $n$.

- for every $n \in\{1, \cdots, N\}, \mathbf{p}^{n} \in \mathbb{R}^{L}, \mathbf{p r c v}^{n} \in \mathbb{R}^{L}$ are the vectors of transmitted and received powers allocated on links in time slot $n$, respectively.

- $\mathbf{P}^{M A X} \in \mathbb{R}^{L}$ is the vector of maximum allowed transmission powers on links, which are assumed constant in time (every link may have a different maximum power).

- $\eta \in \mathbb{R}$ is the white noise at a receiver, and is assumed constant for all links in time.

- for every $n \in\{1, \cdots, N\}$, $\mathbf{S N R}^{n} \in \mathbb{R}^{L}$ is the vector of signal-to-noise ratios at the links' receivers in time slot $n$.

- for every $n \in\{1, \cdots, N\}, \alpha^{n} \in[0,1]$ is the relative frequency of time slot $n$ in the schedule.
- $R$ (routing matrix) is such that $R_{l, i}=1$ if fbw $i$ uses link $l$. We have $\mathbf{f} \leq R \overline{\mathbf{x}}$. The matrix $R$ is defined by the routing algorithm.

- $h_{l_{1} l_{2}}$ is the attenuation of a signal from the source of link $l_{1}$ to the destination of link $l_{2}$.

- $C_{r}$ and $r_{i}$ are the overhead of routing and the number of hops of fbw $i$ respectively, as described in Section III-D.

- $q$ is the probability of loosing a packet due to mobility, as described in Section III-E.

- $\mathbf{f}^{\mathrm{nr}} \in \mathbb{R}^{I}, \mathbf{f}^{\mathrm{nm}} \in \mathbb{R}^{I}, \mathbf{f}^{\mathrm{nrm}} \in \mathbb{R}^{I}$ are the vector of average rates achieved by fbws, when the overheads of routing $\left(C_{r}=0\right)$, mobility $(q=0)$, and both mobility and routing $\left(C_{r}=0, q=0\right)$ are zero, respectively.

\section{B. Physical Network Model}

In this section we describe in details the ultra-wide band physical model of the network on which we base the design of our protocol. We first present a brief description of the WinScholtz physical model [33] which we use latter as an example to illustrate our modeling assumptions. However, we recall that our model is valid for large variety of UWB physical layers.

1) The Win-Scholtz Physical Model: We assume that the physical layer is based on the ultra-wide band radio described in [33]. This radio is based on pulse position modulation (PPM) and a coherent receiver.

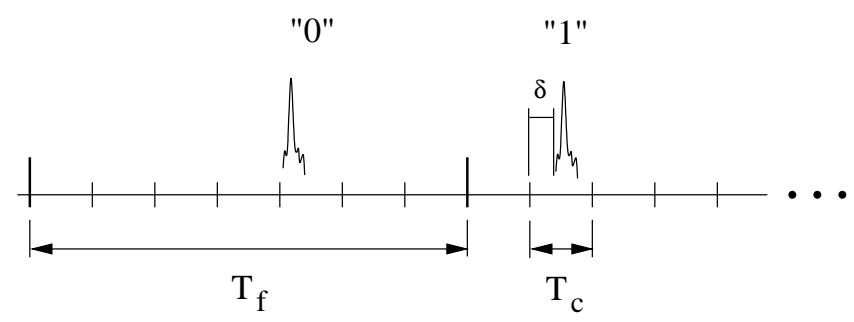

Fig. 1. Ultra-wide band physical layer with PPM, the model of Win Scholtz [33].

Time is divided in frames of duration $T_{f}$. Each frame is divided into bins of duration $T_{c}$. A node transmits one pulse per frame, and it has a pseudo-random time hopping sequence that tells it in which bin to transmit. A time hopping code for the example on the Fig. 1 is $\{5,2, \ldots\}$. Having chosen a bin, the node sends a very short pulse within the duration of the bin. If it is sending a logical zero, this pulse is sent at the beginning of the bin, and if it is sending a logical one, the pulse is delayed by $\delta$. On the example on Fig. 1, the first pulse carries zero, and the second carries one.

Time hopping is used to achieve multiple access. The source and the destination of each link have a common pseudorandom time hopping sequence that is independent of other links' sequences. For other users not knowing the time hopping sequence, this signal has the statistical properties of Gaussian noise, due to randomness in time-hopping codes. It has been shown that for the particular receiver used in [33], the total noise received, comprised of background noise and a sum of signals from other active links, will be perceived by the decoder as a Gaussian noise. 
2) Power Attenuation: Assume the source of link $l$ sends data in a given time slot $n$ with transmission power $p_{l}^{n}$. The power of a signal at the receiver is attenuated due to wave propagation and is $p_{\operatorname{rcv}_{l}^{n}}^{n}=p_{l}^{n} h_{l l}$. We also assume there is no fast random fading, since this has been shown in [28], [25] for wide-band radios. Slow random fading, which is due to mobility, is typically of a larger time-scale than a packet transmission. The mobility thus does not affect packet reception, since channel can be estimated after each packet, but it affects a routing protocol, as explained in Section III-E.

We further assume that a transmitted signal attenuates with power law as a function of distance from the sender. If the link length is $d_{l}$ then we have $h_{l l}=K_{a} d_{l}^{-\gamma}$, and the strength of the signal at the receiver $p_{\mathrm{rcv}_{l}}^{n}$ will be

$$
p_{\operatorname{rcv}_{l}^{n}}^{n}=p_{l}^{n} K_{a} d_{l}^{-\gamma}
$$

where $K_{a}$ and $\gamma$ are constants. This is a commonly used attenuation model for wireless transmissions, and has been confirmed as an indoor propagation model for UWB in [9]. We assume further on $K_{a}=1$ since the parameter $K_{a}$ influences the results only to a constant factor.

3) Rate Function: Let $E_{x}$ be the average received power of a symbol and let $\eta$ be the average power of the noise at the receiver of link $l$ during time slot $n$. This noise consists of a white noise plus the total interference from other users. We approximate this noise as Gaussian. Although it is not always true, the Gaussian approximation holds in most of the cases; it has been shown in [33] that it holds for Win-Scholtz UWB physical model in presence of a large number of interferers. The signal-to-noise (SNR) ratio at the receiver is then $\mathrm{SNR}_{l}^{n}=$ $E_{x} / \eta$.

Information-theoretic results in [31], [28] show that a Shannon-capacity of a multi-path fading AWGN wide-band channel is a linear function of SNR

$$
x_{l}^{n}=K \times \mathrm{SNR}_{l}^{n} .
$$

Thus for a given desired bit-error rate on the link, an efficient wide-band physical layer implementation should have a linear rate function within the operational interval of SNRs.

Equation 2 can be easily demonstrated on a widely known UWB model of Win-Scholtz [33], which is based on PPM and time-hopping. From the Eq. 8 in [6], we see that when we fix the desired bit-error rate $P(e)$ we have that $K^{\prime}=N_{s} * E_{x} / \eta$. Since $\mathrm{SNR}=E_{x} / \eta$ is the signal-to-noise ratio of the received symbol, and the rate $x_{l}^{n}=1 / N_{s} T_{f}$, this yields straightforward to (2), with $K=1 / K^{\prime} T_{f}$ being a function of bit-error rate and the length of time-hopping sequences.

The key difference between narrow-band and wide-band physical layers is that narrow-band ones have a strictly sublinear (typically log) rate function. Most of our findings hold only for a linear rate function. For more discussion see Section VIII.

The key difference between this models and the 802.11 LAN standard as modeled in [11] is that 802.11 allows only limited interference. On one hand, if the interference is high and the signal power is low, so that the SNR is below the threshold, 802.11 will not be able to receive any data. On the other hand, we assume our physical model can adapt its coding (either by using convolutional coding [12], [8] or simple repetition coding [33]) and still achieve positive rate, for arbitrary SNR value. We show later that these differences lead to a fundamental change in MAC protocol design. In the performance analysis throughout the paper we always use our model of the physical layer, in order to allow for a fair comparison of MAC and routing protocols.

4) Orthogonality Factor: It has been shown in [24] that for very large bandwidth parallel transmissions become completely orthogonal and do not interfere with each other. However, in the case of finite bandwidth system it is never the case. We introduce an orthogonality factor $\beta$ that models how much of wide-band interference is captured by a receiver. The specific value of the orthogonailty factor depends on the implementation of a UWB system. In the case of Win Scholtz [33] model, as shown in [6], this factor is of the order of $1 / T_{f}$.

If $\eta$ is the white noise at a receiver, and $Z$ is the total interference from other sources, then the effective noise observed through the decoding process is $\eta+\beta Z$ and the rate of link $l$ in slot $n$ is

$$
x_{l}^{n}=K \times \frac{p_{l}^{n} h_{l l}}{\eta+\beta Z}=\frac{K}{\beta} \times \frac{p_{l}^{n} h_{l l}}{\eta / \beta+Z} .
$$

We see that the optimal architecture of such a network with white noise of intensity $\eta$ is thus equivalent to the same network without orthogonality factor, but with white noise intensity $\eta / \beta$. Thus, without loss of generality, we can assume that $\beta=1$.

5) Power Constraints: In our generic UWB physical layer model, data are sent in packets, and the rate of each packet is given by (2), which is a function of the average packet sending power $p_{l}^{n}$ of link $l$ in slot $n$. The average packet sending power $p_{l}^{n}$ is the average power of symbols in the codebook used in slot $n$, and is limited by

$$
p_{l}^{n} \leq P_{l}^{M A X}
$$

due to various hardware and regulation constraints, and it is assumed constant in time.

We next illustrate how (4) can be derived for a realistic physical model, on the example of Win-Scholtz model [33]. We consider two types of limits imposed by hardware implementations and regulations: pulse power limit $P^{M A X p e a k}$, and average power limit $P^{M A X a v g}$.

Let us denote with $E_{x}$ the energy and with $T_{c}$ the duration of a UWB pulse of link $l$ in slot $n$. Since a pulse is very short, we can assume it has a constant power $E_{x} / T_{c}$. In the model described in [33] a sender is allowed to send only one pulse during the duration of one frame $T_{f}$. The average transmission power during slot $n$ is then $p_{l}^{n}=E_{x} / T_{f}$.

The power of a transmitted UWB pulse is limited by the pulse power limit as $E_{x} / T_{c} \leq P_{l}^{M A X p e a k}$, or $p_{l}^{n} \leq$ $P_{l}^{M A X \text { peak }} T_{c} / T_{f}$. The average power limit imposes $p_{l}^{n} \leq$ $P_{l}^{M A X a v g}$. In the Win Scholtz model, it is assumed that the duration of a frame $T_{f}$ and a chip $T_{c}$ are fixed and predefined by a protocol. Therefore, the only free parameter in the model is the average power $p_{l}^{n}$, and we have a single power constraint 


$$
\begin{aligned}
& P_{l}^{M A X}: \\
& \quad p_{l}^{n} \leq P_{l}^{M A X}=\min \left\{P_{l}^{M A X p e a k} \frac{T_{c}}{T_{f}}, P_{l}^{M A X a v g}\right\} .
\end{aligned}
$$

\section{MAC Layer}

The MAC layer of a network defines power control and scheduling policies. We assume that time is divided into time slots of arbitrary lengths. In each time slot each node can choose to transmit with an arbitrary power that is constant throughout the slot and constrained by the link's power limitations, or it can choose to remain silent. A node cannot send and receive within the same slot, nor it can send or receive from two nodes at a time. A schedule defines in which slot a node transmits, to whom, and with what power. Although a schedule can have any number of slots, we show in Section IV-A that it is sufficient to consider only schedules with a bounded number of slots. Each slot $n$ is characterized by its power allocation $\mathbf{p}^{n}$ and its relative frequency $\alpha^{n}$, which represents what fraction of the overall schedule is occupied by that slot. We do not need to specify the time-scale of a schedule, but we assume it is sufficiently larger than the UWB symbol duration. A slot can be scheduled with different durations in arbitrary time intervals, as long as it relative frequency remains unchanged.

One example of schedule is to allow only a single node to send at any point in time. We call it "total exclusion"; it can be implemented with time division multiple access (TDMA) or contention resolution protocols such as CSMA or token passing. Such a protocol is proposed in the 802.15.3a standard. Another example is a schedule in which as many nodes as possible send at the same time, as in [25].

Protocols implementing a given scheduling scheme usually involve a large complexity, and are left outside the scope of this paper. Also, the implementation of a MAC layer typically includes ARQ related retransmission schemes. By using equation (2), which describes the Shannon capacity, we implicitly assume that the MAC layer uses FEC and ARQ such that they approach the capacity. An efficient implementation of these schemes is also out of scope of this paper.

\section{Routing}

Communication is possible simply by letting each source transmit data directly to its destination. We call this direct routing (DIR). If all nodes use direct routing, we essentially have a single-hop network. The key question is whether one can improve performance by relaying data over intermediate nodes.

In a wireless setting each node can communicate to every other node, and there is an exponential number of possible relaying paths. Additionally, each source can use several paths in parallel, depending on the load on each of them, in order to achieve Wardrop equilibrium [17]. These paths depend on rates and routes of other fbws, and are not necessarily close to the shortest path.

Finding the optimal routing that achieves Wardrop equilibrium is a complex task, and most of the existing multi-hop wireless networks restrict choosing routes from a predefined subset of routes, for example a subset of routes in which each hop decreases the distance to the destination (like AODV or DSR).

In this paper we focus on relaying over nodes that are on the minimum energy and loss route (MELR). If we consider a network with only one fbw, where there can be at most one node sending at a time, then the minimum energy and loss route is the route of the fbw that maximizes its rate. If the network is static and has uniform power constraint, the MELR route is equivalent to the minimum energy route (MER). For more precise definitions of MELR and MER, see Section IVC.

We assume all nodes have the same routing policy. The policy is defined by the length of the hop. One example of such a route is the MELR route itself, where we use the largest number of hops and the smallest hop lengths. By increasing hop length, thus taking a smaller subset of MELR route, we can obtain intermediate paths, as shown on fig. 2. Finally, for very large hop lengths we obtain the DIR routing policy.

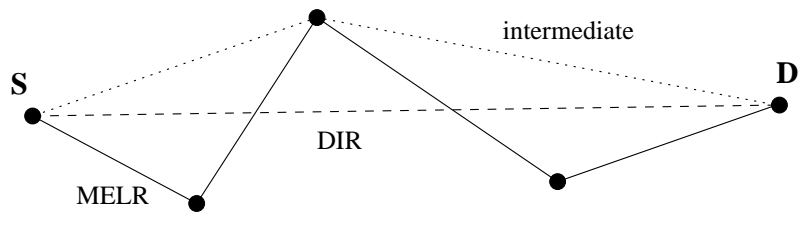

Fig. 2. Different routing policies: DIR sends data directly from a source to a destination (dashed line); MELR relays over nodes that are on a minimum energy and loss path (solid line); intermediate routes use larger hops and relay over a subset of MELR nodes (dotted line).

Whatever routing protocol a wireless network utilizes, it will always waste some rate on the protocol overhead, such as beaconing. This in turn decreases the efficiency of a network that implements routing. We assume that each link consumes a fraction $C_{r}$ of its available rate on routing, hence if the rate of a fbw $i$ is $f_{i} \mathrm{nmr}$ without routing overhead, then the rate of the fbw is $f_{i}^{\mathrm{nm}}=\left(1-C_{r}\right) f_{i}^{\mathrm{nmr}}$ when routing is turned on (this assumes no mobility; mobility is discussed in the following subsection). In order to assess the cost of routing, we also consider a scenario without routing where a source always sends data directly to the destination. We analyze both scenarios in order to estimate what is the acceptable cost of a routing protocol in terms of rate overhead, if it is to be implemented in a network.

In the model of UWB physical layer we assume that the rate is a linear function of SNR. As mentioned in Section III-B.3, this is true only in the operational limit of the physical layer, and can be violated if the link is too long. If a link is long and the SNR at the receiver is below the received threshold, the physical layer operates in very suboptimal region (see for example [33], [25]). If the attenuation on a link is too large, we assume that the link does not exist and we do not consider it in the routing protocol.

\section{E. Mobility}

We assume all nodes are mobile. Although we do not explicitly model nodes' movements, we describe their mobility 
in our model through a packet loss probability at every hop. If a node moves away, a route is broken and packets are lost. When a new node arrives, the route is reconciled, and from there on packets are transmitted. The more the nodes are mobile, the more the packets will be lost due to route destructions.

In our model we consider a snapshot of a network where nodes are not moving. This represents a "typical" topology. Let us denote with $q$ the probability of loosing a packet at a node, or a fraction of packets that will on average be lost at each node on a long term, due to mobility. We model the mobility through $q$ : the higher $q$ is, the more the nodes are mobile. Assume that the rate of fbw $i$ when nodes are not mobile is $f_{i}^{\mathrm{nm}}$, and the number of hops on the route is $r_{i}$. Then, the rate of the same fbw when nodes are mobile is $f_{i}=f_{i}^{\mathrm{nm}} \times(1-q)^{r_{i}-1}$.

The mobility model considered here is on a very highlevel, and might be very inaccurate if compared to more realistic models. However, a more realistic mobility model requires details of a routing protocol implementation. In this paper we focus on the guidelines for designing the optimal protocols, and not on the actual protocol implementation details, therefore a more detailed analysis of the inftuence of different mobility models is out of scope of the paper.

\section{F. Traffic Demand and Flow Control}

We assume all fbws have infinite amounts of data to send. Since lower protocol layers will define in a unique way the available rate for each fbw, we assume our fbw control layer is able to completely use this available rate.

\section{G. Performance Objectives}

As explained in Section I-D, our performance objective is to find a scheduling, routing and power allocation such that the long term average fbw rates are proportionally fair:

Definition 1: Rate allocation $\mathbf{f}$ is proportionally fair [17] on set of rates $\mathcal{F}$ if it maximizes $\sum \log \left(f_{i}\right)$ on set $\mathcal{F}$.

Proportional fairness is also called a utility based metric: each fbw is assigned a utility function $U(f)=\log (f)$, where $f$ is the rate of the fbw. Proportionally fair rate allocation is the one that maximizes the total utility of the network, which is equal to the sum of the utilities of all fbws. Finding the proportionally fair rate allocation is the optimization problem that maximizes the total utility of the network. It is known to have a unique solution.

\section{Mathematical Analysis of the Model}

\section{A. Mathematical Formulation}

We assume that a schedule consists of time slots $n=$ $1 \ldots N$ of frequency $\alpha_{n}$. We normalize these lengths such that $\sum_{n=1}^{N} \alpha_{n}=1$. Let us call $\mathbf{p}^{n}$ the vector of transmission powers assigned to links in slot $n$, and let $\mathbf{S N R}^{n}$ be the vector of signal-to-noise ratios at receivers of the links, induced by $\mathbf{p}^{n}$. The rate achievable on link $l$ in slot $n$ is $\mathbf{x}_{l}^{n}=$ $K \mathbf{S N R}_{l}^{n}$. The vector of average rates on the links is thus $\overline{\mathbf{x}}=\sum_{n=1}^{N} \alpha_{n} \mathbf{x}^{n}$. Since $\mathbf{x}^{n}$ has dimension $L$ (where $L$ is a number of links), by virtue of Carathéodory theorem, it is enough to consider $N \leq L+1$ time slots of arbitrary lengths $\alpha$ in order to achieve any point in the convex closure of points $\mathbf{x}^{n}$.

We next describe set $\mathcal{F}(R)$ of feasible average fbw rates under a given routing matrix $R$. It is the set of $\mathbf{f} \in \mathbb{R}^{I}$ such that there exist a schedule $\alpha$, a set of power allocations $\mathbf{p}^{n}$ and a corresponding set of rate allocations $\mathbf{x}^{n}$ for all $n=$ $1 \cdots N$, and average rates $\overline{\mathbf{x}}$, such that the following set of equalities and inequalities are satisfied for all $n=1 \cdots N, i=$ $1 \cdots I, l=1 \cdots L, o=1 \cdots O:$

$$
\begin{aligned}
f_{i} & =f_{i}^{\mathrm{nmr}}\left(1-C_{r}\right)(1-q)^{r_{i}-1} \\
\mathbf{f}^{\mathrm{nmr}} & \leq R \overline{\mathbf{x}} \\
\overline{\mathbf{x}} & =\sum_{n=1}^{L+1} \alpha_{n} \mathbf{x}^{n} \\
\mathbf{x}_{l}^{n} & =K \mathbf{S N R}_{l}\left(\mathbf{p}^{n}\right) \\
\mathbf{S N R}_{l}\left(\mathbf{p}^{n}\right) & =\frac{\mathbf{p}_{l}^{n} h_{l l}}{N+\sum_{k l} \mathbf{p}_{k}^{n} h_{k l}} \\
1 & =\sum_{n=1}^{L+1} \alpha_{n} \\
1 & \geq \sum_{l: l . \mathrm{src}=o} 1_{\left\{p_{l}^{n}>0\right\}} \\
1 & \geq \sum_{l: l . \mathrm{dst}=o} 1_{\left\{p_{l}^{n}>0\right\}} \\
\mathbf{p}_{l}^{n} & \leq P_{l}^{M A X}
\end{aligned}
$$

where $l \cdot \operatorname{src}=o$ and $l$.dst $=o$ are true if node $o$ is the source or the destination of link $l$, respectively.

The goal of the problem is to find the proportionally fair rate allocation on the set of feasible rates. As follows from the definition 1 , it is equivalent to the following optimization problem:

$$
U=\max _{R \in \mathcal{R}, \mathbf{f} \in \mathcal{F}(R)} \sum_{i=1}^{I} \log \left(f_{i}\right)
$$

where $\mathcal{R}$ is the set of possible routing algorithms defined by Section IV-C. The optimization problem (6) has as free variables routing matrix $R$, time slots' frequencies $\alpha_{n}$, and vectors of transmission powers assigned to links in slot $n, \mathbf{p}^{n}$. The values of these variables that solve (6) define the optimal routing, scheduling and power control in the given network.

Definition 2: A tuple $\left(R, \alpha_{n}, \mathbf{p}^{n}\right)$ is called optimal routing, power allocation and scheduling if it solves the optimization problem (6).

\section{B. Optimal Power Allocation}

In this section we provide a characterization of the optimal power allocation. We prove that, regardless of the choice of routing and MAC protocol, the proportionally fair rate allocation can be achieved using power allocations such that each link in every time slot is allocated either zero power or full power.

Seemingly similar findings have been already presented in [4], [5]. However, in those paper, the authors consider the total sum of rates as the performance metric of interest. Although a use of the sum of rate metric facilitates the proofs, this metric is known to be unfair and inappropriate for ad-hoc networks [23]. Furthermore, it is not possible to extend the existing proofs to utility based performance metrics. We generalize 
the statements from [4], [5] by showing that all feasible rate allocations can be achieved using extreme power allocations. We start by defining the notion of achievable rate allocation.

Definition 3: We say that an allocation $\mathbf{f}$ is achievable with a set of power allocations belonging to $\mathcal{P}$ if it satisfies the following set of constraints for all $n=1 \cdots N, i=1 \cdots I, l=$ $1 \cdots L, o=1 \cdots O$ :

$$
\begin{aligned}
f_{i} & =f_{i}^{\mathrm{nmr}}\left(1-C_{r}\right)(1-q)^{r_{i}-1} \\
\mathbf{f}^{\mathrm{nmr}} & \leq R \overline{\mathbf{x}} \\
\overline{\mathbf{x}} & =\sum_{n=1}^{L+1} \alpha_{n} \mathbf{x}^{n} \\
\mathbf{x}_{l}^{n} & =K \mathbf{S N R}_{l}\left(\mathbf{p}^{n}\right) \\
\mathbf{S N R}_{l}\left(\mathbf{p}^{n}\right) & =\frac{\mathbf{p}_{l}^{n} h_{l l}}{N+\sum_{k \neq l} \mathbf{p}_{k}^{n} h_{k l}} \\
1 & =\sum_{n=1}^{L+1} \alpha_{n} \\
1 & \geq \sum_{l: l . \mathrm{src}=o} 1_{\left\{p_{l}^{n}>0\right\}} \\
1 & \geq \sum_{l: l . \mathrm{dst}=o} 1_{\left\{p_{l}^{n}>0\right\}} \\
\mathbf{p}^{n} & \in \mathcal{P}
\end{aligned}
$$

This set of constraints is almost equivalent to the one from (5), except it has additional restrictions that all power allocation used to achieve $\mathbf{f}$ belong to $\mathcal{P}$.

Let us denote with $\mathcal{F}$ the set of feasible fbw rate allocation f described with (5) and let $\mathcal{P}^{E}$ be the set of extreme power allocations $\mathcal{P}^{E}=\left\{\mathbf{p} \mid(\forall l=1 \cdots L) p_{l} \in\left\{0, P_{l}^{M A X}\right\}\right\}$. We further define $\mathcal{F}^{E}$ to be the set of fbw rates $\mathbf{f}$ that can be achieved with set $\mathcal{P}^{E}$, or in other words when the power on each link in every slot $p_{l}^{n}$ is either 0 or $P_{l}^{M A X}$. Since every fbw rate allocation vector from $\mathcal{F}^{E}$ is feasible, we have $\mathcal{F}^{E} \subseteq$ $\mathcal{F}$. We want to show that every feasible fbw rate allocation can be achieved by a set of extreme power allocation from $\mathcal{P}^{E}$, that is $\mathcal{F} \subseteq \mathcal{F}^{E}$.

Theorem 1: For arbitrary values of parameters of constraint set (5), we have $\mathcal{F}=\mathcal{F}^{E}$.

The proof of the theorem is in the Appendix. As a corollary of the theorem, it follows that the proportionally fair rate allocation can be achieved with a set of power allocations that allocate zero or maximum power to each link in every slot.

\section{Minimum Energy and Loss Routes}

We here define the notion of Minimum Energy and Loss Route (MELR), and give an intuitive interpretation.

Definition 4: The Minimum Energy and Loss Route (MELR) of a fbw is the route that minimizes

$$
\sum_{i=1}^{r}\left(P_{i}^{M A X} h_{i}\right)^{-1}(1-q)^{-r+1}
$$

over all possible routes.

Proposition 1: Consider a network depicted with (5) with a single fbw, and let us restrict the scheduling such that there may be, at most, one node sending at a time with maximum power. Then, the optimal routing for the fbw is MELR routing.

Proof: Consider an arbitrary route $\left\{l_{1}, \cdots, l_{r}\right\}$ from the source to the destination and let $h_{i}$ be the fading on the $i$-th link. In the optimal rate allocation we have rates of all links equal to the rate of fbw. Due to the restriction in the schedule, a rate of link $i$ is $f=\bar{x}_{i}=\alpha_{i} K P_{i}^{M A X} h_{i} / N$, where $\alpha_{i}$ is the fraction of time link $i$ is scheduled. Again, in the optimal allocation we have $\sum_{i=1}^{r} \alpha_{i}=1$. From the above equation it is easy to derive that

$$
f=K\left(N \sum_{i=1}^{r} \frac{1}{P_{i}^{M A X} h_{i}}\right)^{-1}(1-q)^{r-1} .
$$

From there we see that a path that maximizes the rate in the network minimizes $\sum_{i=1}^{r}\left(P_{i}^{M A X} h_{i}\right)^{-1}(1-q)^{-r+1}$, which is indeed the MELR.

In the case of a static network with uniform power constraints, the MELR route is equivalent to the well known energy route (MER), which we recall below.

Definition 5: Suppose a path $r$ consists of nodes $r(1), \cdots, r(n)$, and let us denote with $h_{i, j}$ the attenuation between nodes $i$ and $j$. The minimum energy route (MER) of a fbw is the one that minimizes

$$
\sum_{i=1}^{n-1} h_{r(i), r(i+1)}^{-1}
$$

over all possible routes $r$ for the fbw.

Furthermore, if we consider the scenario from proposition 1, where the network is static and has uniform power constraints, and we fix the rate of the fbw, then the MER route is indeed the one to minimize the dissipated energy.

\section{Techniques for Solving the Problem}

Even once we have successfully characterized the optimal power allocation, the considered optimization problem remains a highly complex optimization problem, and is difficult to solve in the general case, even using advanced optimization methods such as in [15]. A discussion for arbitrary networks with up to 6 nodes is given in [29]. It is difficult to draw general conclusions about network design from such small networks. Our method to overcome this is as follows.

First, we solve exactly a small, one-dimensional simple network. We consider a ring topology where all nodes are equally spaced and send data to their $d$ hop away neighbor on the right. We are able to find an analytical solution for this simple model. This initial steps allowed us to identify the properties of the optimal solution that we describe as findings $1,3,4$ and 5 in Section I-G.

Then we next consider a random, two-dimensional network. We define a number of alternative strategies for scheduling and routing, and analyze the performance of the various combinations. The routing strategy decides how many nodes along the MELR route is spanned by one hop. The scheduling policy is one of the following four: all nodes at a time (interference is always allowed), only one node at a time (this is, in our framework, equivalent to time division multiple access, TDMA), and two strategies with exclusion around the destination. The last two strategies work as follows. Having chosen a route, each node selects a blocking distance, that is the radius of a disk around the receiving node in which there must be no other active nodes. We repeat the same procedure 
for each node and each fbw, and then construct a schedule in a greedy manner. Then, we optimize slot frequencies in order to maximize system utility. The two strategies with exclusion differ in the way they compute the blocking radius. One optimizes the radius based on local information: next hop distance, available power and position of surrounding nodes. The other computes the radius based solely on the transmission power of the source of the link.

We numerically analyze the performance of these strategies on a set of networks with up to 50 nodes, where nodes are randomly distributed. Since UWB propagation is short range, we expect such network sizes to be realistic. We consider uniform and non-uniform network topologies, both in the sense of node positioning, power constraints and traffic demands. We first consider a network of uniformly distributed nodes on a unit square, where half of the nodes are sources talking to a randomly chosen destinations from the other half of the nodes. We next consider heterogeneous scenarios where randomly distributed nodes all talk to a few base stations. Finally, we consider networks with non-uniform node distributions where, again, half of the nodes talk to the other half. We further consider different levels of mobility, as this implies unreliable routes and incurs additional cost to routing. We also consider networks whose nodes have non-uniform power constraints, which model different types of wireless equipment in the same network - from high-power laptops to small-power ubiquitous computing devices.

\section{The Static Ring Case}

As mentioned earlier, the above problem is difficult to solve in the general case. In order to obtain heuristics that will help us find an approximate solution for a general problem, we first restrict our attention to a static network with ring topology and with a high level of symmetry, as depicted on Fig. 3. In order to maintain symmetry of the network, we also assume there is no mobility $(q=0)$, hence there is no routing overhead $\left(C_{r}=0\right)$. Arbitrary mobile networks in plane are discussed later in Section VI.

The ring topology can be represented as an $L$-sided regular polygon with distance $l$ between nodes.

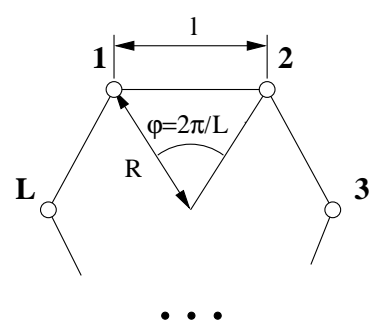

Fig. 3. Analyzed topologies: ring.

The maximum power for all nodes is the same, and equal to $P$. Each node is a source of data, and its destination is $d$-hops away on the right, where $d<L / 2$, thus here $I=L$.

Next we present several propositions related to the ring topologies. We prove them, and use them to simplify the numerical solution of the optimization. We then define several claims and we verify them by inspecting the numerical results on various ring sizes. These findings are then extended and verified to arbitrary networks in Sections VI and VII.

\section{A. Proportional Fairness}

The first proposition is about a property of the proportionally fair rate allocation.

Proposition 2: In the above defined ring wireless network, if a rate allocation is proportionally fair then each fbw has the same rate.

Proof: We proceed by contradiction. Let us denote with $\mathbf{f}^{*}$ the proportionally fair rate allocation and assume that for some $i, j, f_{i}^{*} \neq f_{j}^{*}$. Let $\left(R, \alpha_{n}, \mathbf{p}^{n}\right)$ be the optimal routing, scheduling and power allocation that achieves $\mathbf{f}^{*}$.

By time division and rotation of the optimal allocation, it is possible to achieve rate allocation $\mathbf{f}$ such that

$$
f_{i}=\frac{1}{L} \sum_{j=1}^{L} f_{j}^{*}
$$

By construction, we have that for all $i, j, f_{i}=f_{j}$, thus we have $\mathbf{f}^{*} \neq \mathbf{f}$.

Finally, by concavity property of the log function we further have that

$$
\log \left(f_{i}\right)=\log \left(\frac{1}{L} \sum_{j=1}^{L} f_{j}^{*}\right)>\frac{1}{L} \sum_{j=1}^{L} \log \left(f_{j}^{*}\right),
$$

which leads to contradiction.

Since all fbw rates are the same, we can write $f=f_{i}$. Due to symmetry in routing, one can easily verify that all link rates are the same, hence we can also write $\bar{x}_{i}=\bar{x}$. For the direct routing, a link capacity corresponds to the rate of a fbw, hence the rate of fbw $f=\bar{x}$. For the minimum energy routing we have $d$ fbws sharing the same link, hence $f=\bar{x} / d$.

\section{B. Scheduling}

We next describe the optimal scheduling in the ring case. In the case of MER routing, we have $L$ one-hop links, and in the case of DIR routing we have $L d$-hop links. We show that the optimal schedule consists of rotationally symmetric slots of equal lengths. For $\mathbf{p} \in \mathbb{R}^{L}$ and $n \in\{1, \cdots, L\}$, let us call $\mathcal{O}_{n}(\mathbf{p})$ a rotation of $\mathbf{p}$ such that for all $n \in\{1, \cdots, L\}$,

$$
\left(\mathcal{O}_{n}(\mathbf{p})\right)_{j}=\mathbf{p}_{[(j+n-1) \bmod L]+1} .
$$

Proposition 3: In the above depicted ring scenario, there exists a power vector $\mathbf{p} \in \mathbb{R}^{L}$ such that the optimal schedule consists of $L$ rotationally symmetric power vectors $\mathbf{p}^{n}=$ $\mathcal{O}_{n}(\mathbf{p})$ that are equally frequent, that is $\alpha_{n}=1 / L$.

Proof: Let $\overline{\mathbf{x}}$ be the vector of the optimal link rates. From proposition 2 it follows that all links have the same rate, that is for all $i$ and $j,(\overline{\mathbf{x}})_{i}=(\overline{\mathbf{x}})_{j}$.

Since $\overline{\mathbf{x}}$ is rotationally symmetric we can achieve the same rate by rotating each power allocation by an arbitrary $k$, hence from (5):

$$
\overline{\mathbf{x}}=\sum_{n=1}^{L+1} \alpha_{n} K \operatorname{SNR}\left(\mathcal{O}_{k}\left(\mathbf{p}^{n}\right)\right) .
$$


It follows that $\mathbf{x}^{n}=1 / L \sum_{k=1}^{L} K \operatorname{SNR}\left(\mathcal{O}_{k}\left(\mathbf{p}^{n}\right)\right)$, thus all $\mathbf{x}^{n}$ are also rotationally symmetric. Since

$$
\overline{\mathbf{x}}=\sum_{n=1}^{L+1} \alpha_{n} \mathbf{x}^{n} \leq \max _{n} \mathbf{x}^{n},
$$

we conclude that for some $\mathbf{p}$ we can represent the proportionally fair allocation as

$$
\overline{\mathbf{x}}=\frac{1}{L} \sum_{n=1}^{L} K \mathbf{S N R}\left(\mathcal{O}_{n}(\mathbf{p})\right) .
$$

\section{Power Allocation}

Proposition 4: In the optimal power allocation in a ring network, each node in a given time either sends at the maximum power or does not send at all.

The proposition is a direct consequence of Theorem 1, and it proves our finding 3, that all nodes should send at the maximum power when sending. Note that the result of Theorem 1 is applicable to an arbitrary network, and not only a ring network, as stated in Proposition 7.

\section{Routing}

At this point we analyze the optimal routing policy. As already explained, we assume node $i$ can relay over all nodes on the closest path between $i$ and $d+i$, and the routing policy is defined by the length of the hop. If the length of the hop is 1 , then data is relayed over all nodes between $i$ and $d+i$ and this is the MER, and since the network is static, it is also the MELR route. If the length of the hop is $d$ then the source sends directly to the destination, and the routing is called the direct routing (DIR).

Proposition 5: For any network size, fbw length and maximum power constraint, and under optimal scheduling and power allocation, it is the optimal to use the smallest hop length (i.e. to use the minimum energy route).

We were not able to prove the proposition analytically. However, we solved numerically the optimization problem (5) for rings of various sizes and various fbw lengths, and we found that MER always gave the best performance. An example of comparison between MER and DIR routes can be seen on the top of Fig. 4 for a ring of 14 nodes, and fbws of length 6 .

Proposition 5 confirms our finding 4 for the ring case, that the optimal routing is the MELR routing.

\section{E. Exclusion Regions}

Proposition 6: In the optimal power allocation $\mathbf{p}$, each receiving node has an exclusion region around it in which all nodes are silent. The sizes of the exclusion regions of any two nodes differ by at most one. This is true regardless of the choice of the routing protocol.

The proposition is illustrated on Fig. 5 and Fig. 6. Again, we were not able to prove it analytically, but we tested it for various ring sizes and fbw lengths. This proposition verifies
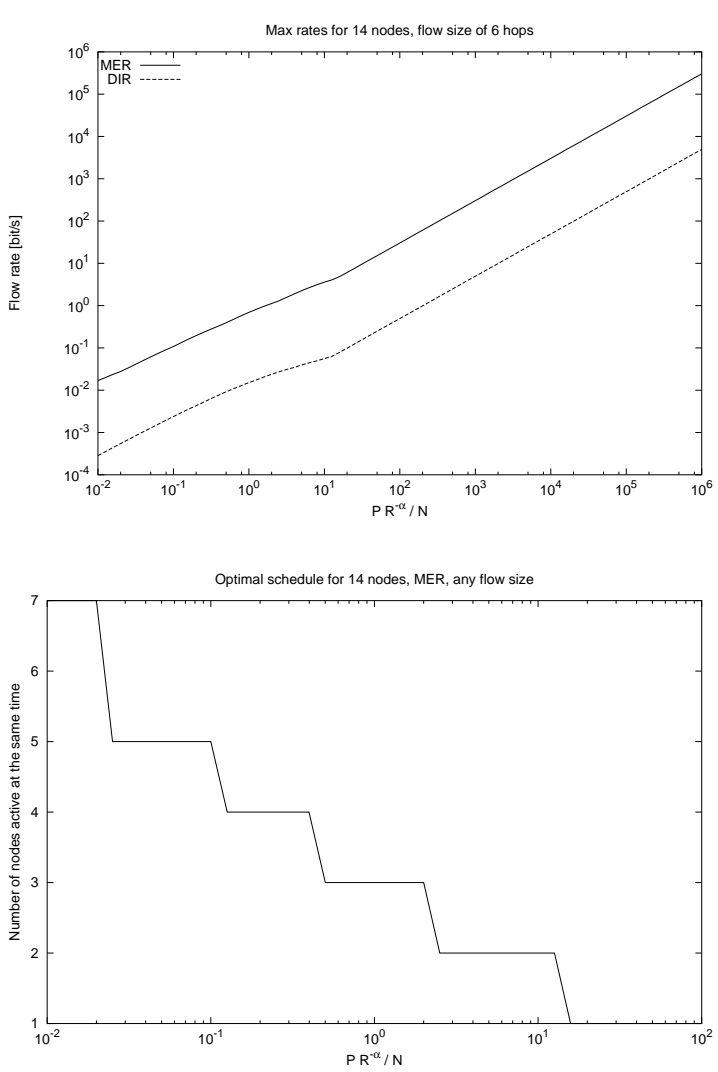

Fig. 4. Top: maximum rates for DIR and MER routing and different values of $d$ are depicted, for a ring of 14 nodes, versus normalized power. We see that MER routing is better then DIR for all transmitting powers constraints. Bottom: we see the optimal number of nodes active in the same slot. For low powers, 7 out of 14 nodes are active at the same time (every second node is sending). For high powers, only one node is sending at a time.

our findings 1 and 5 for the ring case. It also verifies finding 2 , since we show the sizes of exclusion regions differ in at most one.

On the bottom of Fig. 4 one can see how the number of active nodes depend on the relative transmission power $\mathrm{Pl}^{-\alpha} / N$, for MER routing (which is the same as MELR in this case), where $l$ is the distance between two adjacent nodes in the ring, and $N$ is the intensity of the background noise. Whereas for small powers, every second node is active, for large powers only one node is active at a time. We also see that this schedule is independent of the fbw length. The same holds for DIR routing.

\section{Mobile Networks in A Plane}

In this section we solve the optimization problem (6) for an arbitrary network on a plane. Due to the complexity of the problem we are not able to fully solve it theoretically or numerically. We are able to completely characterize the optimal power allocation and give a proof of its optimality. In order to find approximate solutions for the optimal scheduling and routing, we do not explore the full state space, but we use heuristics to find an approximate solution. We apply findings from the symmetric ring case in order to derive the heuristics. We compare these heuristics numerically on a large number 


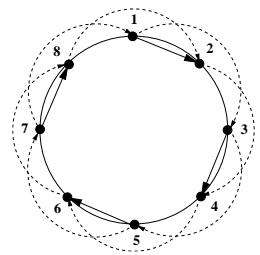

1

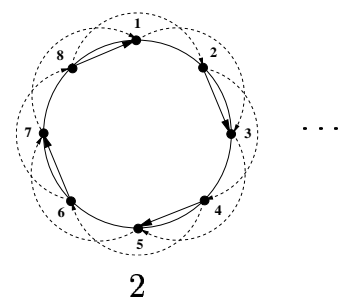

$L=8, d=2, P^{M A X}=10^{-2}$
Fig. 5. Illustration of proposition 6. On the top, the optimal scheduling for MER routing is given, for $L=8, d=2, P^{M A X}=10^{-2}$. Dashed arrows depict flows, and solid arrows depict active links. Numbers below are time slot numbers. Each link is either inactive, or active at the full power. There are 8 allocations in total, but the first 2 are exactly the same as the last 6 . These 8 allocation repeat in a row, each taking the equal time slot, and each one is a rotation of the previous one. The distance $s$ between active nodes is 2 .

$$
L=8, d=2, P^{M A X}=10^{-2}
$$

\begin{tabular}{|c|c|}
\hline MER & DIR \\
\hline 10101010 & 11001100 \\
\hline
\end{tabular}

$$
L=18, d=2, P^{M A X}=10^{-1}
$$

\begin{tabular}{|c|c|}
\hline MER & DIR \\
\hline 100010001000100100 & 111000000111000000 \\
\hline
\end{tabular}

Fig. 6. Short representations of the optimal policies for $L=8$ and $L=18$ : one rotation of the optimal power allocation is given, each link is denoted with 0 if inactive and 1 if active. The upper case $n=8$ is the one depicted on Fig. 5. In MER routing, the size of the exclusion region is smaller than the length of the hop, hence every second node is active. In DIR routing, for $L=8$ there are 2 groups of 2 active nodes, and distance between them is 4 . Again, the size of the exclusion region is smaller than the length of the hop. For $L=18$, and MER routing, the size of the exclusion region is between 2 and 3 hop lengths $l$. In the case of DIR routing, some receivers have the size of exclusion region between $1 l$ and $2 l$, and some between $2 l$ and $3 l$.

of random networks in order to find the approximate solution to the problem (6).

We first analyze the optimal power allocation and we have the following proposition:

Proposition 7: In the optimal power allocation for an arbitrary network in plane, each node in a given time either sends at the maximum power or does not send at all.

The proposition is a direct consequence of Theorem 1, and it proves our finding 3: all nodes should send at the maximum power when sending.

In order to find the optimal routing and scheduling, we propose a number of routing and scheduling strategies. We performed a full factorial analysis by combining these strategies, and we solved the optimization problem (6) for each possible combination.

First, we find the optimal route for each fbw according to the chosen routing strategy. Those routes give us routing matrix $R$ and the set of links that have to transmit in our schedule. Next we use the chosen scheduling strategy to obtain the exclusion lists, that is to say, the lists of nodes that have to remain silent while each destination is receiving.

We assign each link a weight that corresponds to the number of slots in the schedule in which it has to be active and we then use a greedy algorithm to construct a schedule. At this point, a slot represents a set of nodes that will be scheduled at the same time. In the first slot we schedule a random link, and we continue adding random links as long as they do not belong to the exclusion lists of the already scheduled links. When it is not possible to schedule further links in a given slot, we start a new slot. We repeat the algorithm until all links have been scheduled according to their weights. Note that due to the topology of a network it is possible that some links get scheduled in more slots than their weight determines (i.e. a very distant link that does not interfere with others' transmissions will be scheduled in every slot).

Once a schedule is determined, we optimize the lengths of the slots. If a link is scheduled to transmit in a given slot, according to the power hypothesis this means it sends with the maximum power. At this point we are able to calculate $\mathbf{x}^{n}$ link rates in each slot $n$ from the model (5). Having $\mathbf{x}^{n}$ fixed, we optimize slot lengths $\alpha$, which is a purely convex optimization problem, and can be solved using traditional convex programming.

We tried several heuristics to determine weights of links in constructing the schedule:

- Equal weights: All links have weights 1, and all get schedule approximately equal number of times.

- Traffic weights: Each link is assigned a weight that corresponds to the number of fbws passing over it. This means a links with more fbws passing over it will be scheduled more frequently.

- Lagrangian weights: Solving the convex optimization problem that determines $\alpha$ for a fixed schedule, we obtain shadow prices for each component $\bar{x}_{l}$. We use this shadow price as the weight of a link for generating the next schedule.

We repeat the above described procedure several times for a given network. Due to the randomness in schedule generation, we obtain several different results, and we choose the maximal. In addition, in the case of Lagrangian weights, we update the weights for schedule generation at each run.

We tested different choices of scheduling weights on various random network examples. We found on all tested scenarios that changes in weights bring no difference in results, since the slot frequencies also change to compensate. This shows that the solution of our algorithm is not very sensitive to the choice of scheduling policy. Therefore, we further on used the simplest one, the equal weights strategy.

The optimization algorithm described above is implemented in Matlab, and is used to produce all the results presented in this paper (the code can be found on [19]). 
Next, we present the routing and scheduling strategies considered. Numerical results of the optimization for different networks in a plane are given in Section VII.

\section{A. Routing Strategies}

MELR routing strategy: Each fbw uses the minimum energy and loss route (MELR).

Intermediate routing strategy: Flows use routes consisting of nodes that belong to the MELR path. Hop lengths in meters of all fbws are the same.

MER routing strategy: Each fbw uses minimum energy route (MER).

DIR routing strategy: There is no relaying and each fbw uses the direct link (DIR).

MERL routing strategy is derived from the proposition 1 , where we showed it is optimal for a specific network topology. Intermediate routing strategy represents a behavior of conventional routing protocols for wireless networks and gives a large number of possible routings, including DIR and MERL routes as special cases. MER routing is equivalent to MELR on a static network with uniform power constraints. Otherwise it differs, and might not even be comprised of the same nodes as MELR.

\section{B. Scheduling Strategies}

We have seen from the ring case (Fig. 6) that in the optimal schedule, each link should maintain an exclusion region around its destination while receiving. Other nodes outside of that region might be transmitting during the same slot. We maintain the same approach in the $2 \mathrm{D}$ case, and we define two strategies that calculate the size of the optimal exclusion region $s$ around each destination (see Fig. 7).

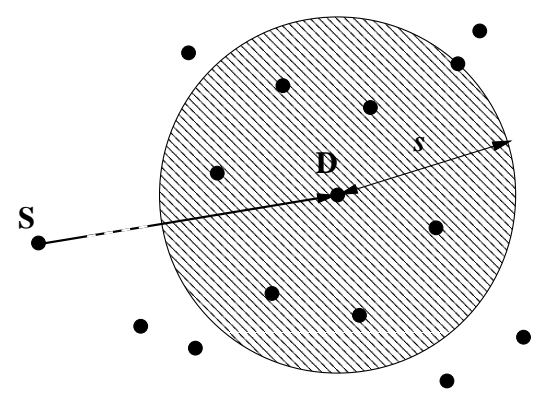

Fig. 7. An example of the exclusion region. Node $D$ is receiving data from node S. Its exclusion region is represented as a shaded circle, and has radius $s$. There are $N(s)$ nodes inside the exclusion region and they are silent during the reception. Nodes outside of the exclusion region can transmit, and the total interference they produce on D is $I(s)$.

Let us consider the destination D of link $l$, and assume it has an exclusion region of size $s$. Let us call $N(s)$ a number of nodes that are in the exclusion region of $\mathrm{D}$, and let us denote with $I(s)$ the interference received by $\mathrm{D}$ if all nodes outside of the exclusion region would be active at the same time at the maximum power. Assume for the moment that nodes are uniformly distributed, and that all receivers have the same size of the exclusion region $s$. Then link $l$ can be scheduled for transmission at approximately every $k_{1} N(s)$ slot. When scheduled, it will experience the interference of approximately $k_{2} I(s)$, where $k_{2}$ models the fact that not all the nodes outside of exclusion region will be scheduled at the time. The rate of link $l$ in that case is

$$
\bar{x}_{l}=\frac{K}{k_{1} N(s)} \frac{P_{l}^{M A X}}{\eta+k_{2} I(s)} .
$$

Based on this equation, we propose the first strategy for finding the size of the exclusion region

Scheduling strategy 1: The receiver of each link maintains an exclusion region while receiving. It calculates $N(s)$ and $I(s)$ and for given $k_{1}$ and $k_{2}$ finds $s$ that maximizes (9). This is the size of the exclusion region. The optimal values of $k_{1}$ and $k_{2}$ are tuned on numerical examples.

Next, we propose a simpler strategy. Again, consider a network with uniformly distributed nodes of density $\lambda$, where all nodes have equal power constraints. Consider the destination D of link $l$. We assume it has the exclusion region of size $s$ and we assume there is a node at the border of this region, at distance $s$ from D. We also assume there is a power law signal attenuation and we approximate $I(s)=P^{M A X} s^{-\gamma}$. We further approximate $N(s)=\lambda s^{2} \pi$, and we look for $s$ that maximizes (9), with $k_{2}=1$. By inspecting the first derivative, we find that the optimal $s$ satisfies

$$
\frac{P^{M A X} s^{-\gamma}}{\eta}=\frac{2}{\gamma-2} \text {. }
$$

Scheduling strategy 2: The receiver of each link maintains an exclusion region while receiving. It calculates the size of the destination region around its destination according to the following formula

$$
s=\left(\frac{(\gamma-2) P^{M A X}}{2 \eta}\right)^{1 / \gamma}
$$

The second strategy strikes with the simplicity of a potential implementation. The left side of (10) represents the SNR of the potential signal a destination would send to announce the exclusion region. Any node that would receive this signal with SNR higher than $2 /(\gamma-2)$ would know that it is in the exclusion region and that it should remain silent.

We also consider Total exclusion and All-at-once scheduling policies for comparison with the two strategies:

Scheduling strategy 3 (Total exclusion): Only one node may send at a time.

Scheduling strategy 4 (All-at-once): As many node as possible can send at a time, as long as each node only sends to or receives from only one node at a time.

Note that these two scheduling policies can be also viewed as policies with exclusion regions. Total exclusion is equivalent to having an exclusion region with the infinite size, while Allat-once is equivalent to having an exclusion region of size 0 .

\section{NUMERICAL RESULTS}

In this section we present numerical solutions of the optimization problem (6) for various network scenarios. The goal of the analysis is to prove our findings 1-5 from Section I-G. 


\section{A. An Example Scenario}

We start by illustrating our results on a simple example, given on Fig 8. The network is static and power constraints are the same for all nodes. This in turn means that MER and MELR routes are equivalent. We compare the total utility (6) achieved by different combinations of routing and scheduling strategies.

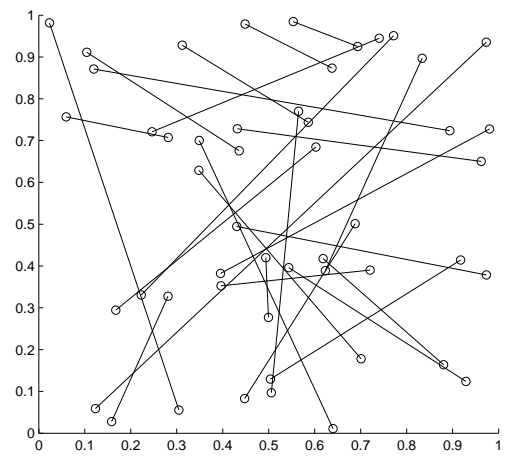

Fig. 8. An example of a network with 50 randomly distributed nodes and 25 randomly distributed flows.

On Fig. 9 we plot the histograms of fbw rates achieved on the network from Fig. 8 with MELR routing and different schedulings. The utility of each allocation is given in the title. We see that the utilities of scheduling strategies 1 and 2 are almost the same. We also see that the utilities achieved by Total exclusion and All-at-once schedulings in this case are significantly smaller. The worse performance in these cases can be verified from the two histograms on the right.

On Fig. 10 we plot histogram of fbw rates achieved on the network from Fig. 8 for DIR and MELR routings, and scheduling strategy 2 . The utility of each allocation is given in the title. We see that MELR routing is in this case significantly better than DIR.
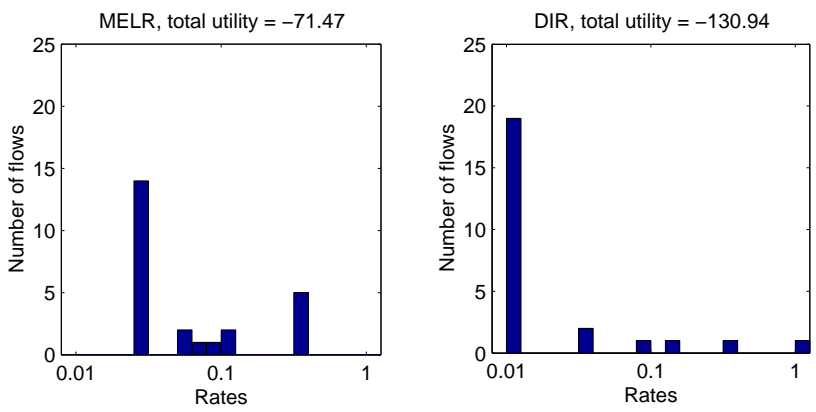

Fig. 10. Histogram of flow rates achieved on the network from Fig. 8 using scheduling strategy 2 and MELR or DIR routing. Maximal transmission power is $P / N=-30 d B$ for all nodes. The total utility achieved is given in the title of each figure. On the $\mathrm{X}$ axis are depicted rates in the log scales. The axis is divided into bins of equal sizes. On the $Y$ axis are the numbers of flows whose rates belong to a given bin.

\section{B. Homogeneous Networks with Homogeneous Traffic}

We first consider homogeneous networks with 50 nodes uniformly distributed on a square of area 1 . Half of the nodes are sources and half are destinations, and each source chooses randomly a destination. An example of such a network is given on Fig. 8.

The main parameter that influences the rate is the received power over noise. Therefore, varying the density of nodes (either by changing the number of nodes or the surface of the area) is equivalent to varying the maximum transmitted power constraint. Also, an increase in the white noise is equivalent to a decrease of maximum transmission power. We consider $P^{M A X} / N$ as the main architecture parameter of our network. We test all possible combinations of routing and scheduling strategies presented in Section VI, for different values of $P^{M A X} / N$.

Value of $P^{M A X} / N$ depends of the system's implementation, and on specific values of the attenuation factors, defined in (1), and the orthogonality factor, defined in (3). For the Win-Scholtz model [33], taking parameters from [6], [9], we obtain $\beta=3.910^{-3}$ and $K_{a}=10^{-5}$ hence the $P^{M A X} / N=$ $-4.62 d B$. The exclusion region size is of the order of several meters. For values of these parameters in a case of a low power UWB system, refer to [18].

An illustration of the comparisons is given on Fig. 11, where we compare MELR and DIR routing combined with all 4 scheduling strategies. We find that the scheduling strategy 2 is the best one, regardless of the routing protocol and the length of hops used. Recall that the size of an exclusion zone in strategy 2 depends on only on the power constraints of the corresponding source, and not on the length of the link or the positions of surrounding nodes. This confirms our finding 1 and 2 about the optimal MAC protocol. It also confirms finding 5 , since neither the scheduling strategy nor the size of the exclusion region itself depend on the choice of the route.

We also compared performances of different routing protocols by varying the length of hops, for different scheduling strategies. An example can be seen on Fig. 11 where we depict performances of DIR and MELR routings for different scheduling strategy. We found that the smallest hop length is the best, and that MELR routing is the optimal routing, regardless of the choice of the scheduling. This confirms our finding 4 .

It is interesting to observe from Fig. 11 that the scheduling strategy 1 , which has more sophisticated procedure of calculating the size of exclusion regions, has a performance equally as good as strategy 2 . We also see that Total exclusion is equally as good as the scheduling strategy 2 for high power constraints, and All-at-once is as good for low power constraints. This is in accordance with the construction of the exclusion region of strategy 2. On one hand, as the power is growing, the exclusion regions are growing as well, until each exclusion region occupies the whole network, and the policy becomes Total exclusion. On the other hand, when power is sufficiently small, exclusion regions will be small enough so that they do not include any nodes, and the policy becomes All-at-once.

\section{Non-Homogeneous Networks}

During the derivations of our strategies we always assumed networks were rather homogeneous: nodes are uniformly distributed, routing matrix is homogeneous, and power constraints 

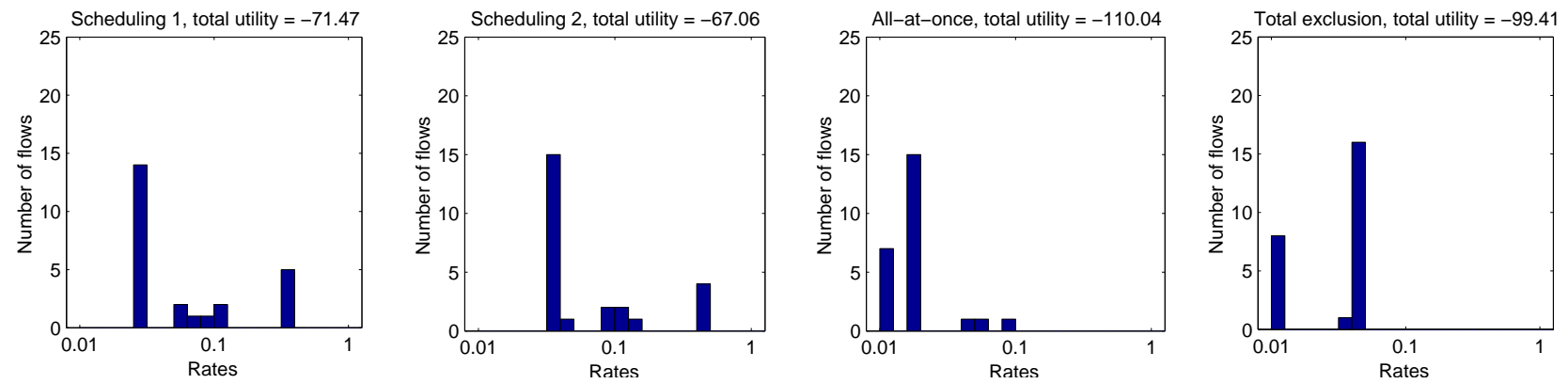

Fig. 9. Histogram of flow rates achieved on the network from Fig. 8 with MELR routing and different schedulings. Maximal transmission power is $P / N=-30 d B$ for all nodes. The name of a strategy with the total utility achieved is given in the title of each figure. On the $\mathrm{X}$ axis are depicted rates in the log scales. The axis is divided into bins of equal sizes. On the $Y$ axis are the numbers of flows whose rates belong to a given bin.
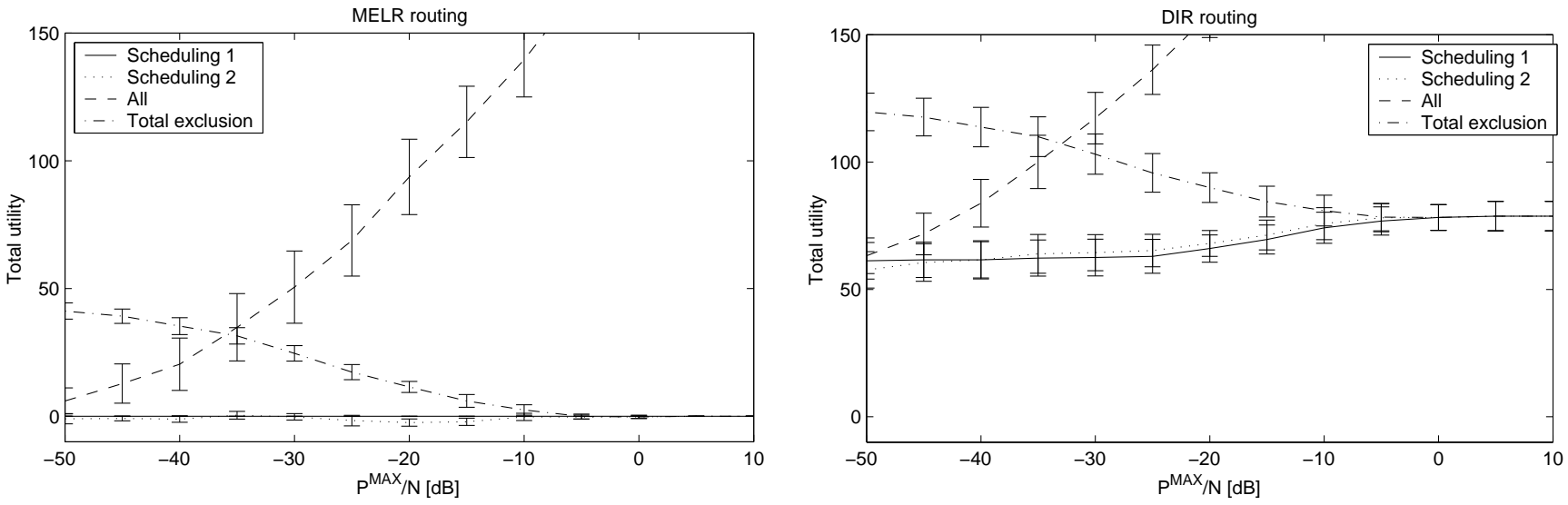

Fig. 11. Utilities of different routing and scheduling algorithms applied on homogeneous random networks and different maximal power constraints. On the left different schedules are applied with MELR routing and on the right with DIR routing. On the $\mathrm{x}$ axis a relative power constraint $P M A X / N$ is given. On the $y$ axis the differences between total utility of the reference approach and the analyzed approach are given. Here, scheduling strategy 2 with MELR is taken as the reference approach.

are the same for all nodes. In this section we analyze if our conclusions are also valid in non-homogeneous networks. We address three types of inhomogeneity: non-homogeneous nodes distribution, non-homogeneous traffic matrix and nonuniform power constraints.

We first consider non-homogeneous node distributions. We now assume that 50 nodes are distributed on the unit square such that 40 nodes are placed uniformly on the left half of the square and the remaining 10 nodes are placed on the right half. Sources and destinations are still chosen uniformly among nodes.

Next, we consider non-uniform traffic matrix. We uniformly place 50 nodes on a unit square, and all of them talk to the nearest base station. We analyzes cases with 1 (placed in the center of the square) and 4 base stations (placed in the centers of four quarters of the square).

Finally, we consider networks with uniformly distributed nodes and source destination pairs, but non-uniform power constraints. Each node has the power constraint randomly chosen from the interval $\left[(1-c) P^{M A X},(1+c) P^{M A X}\right]$. This way the average power constraint of all nodes is still $P^{M A X}$.

In all those cases, we analyze all combinations of schedul-

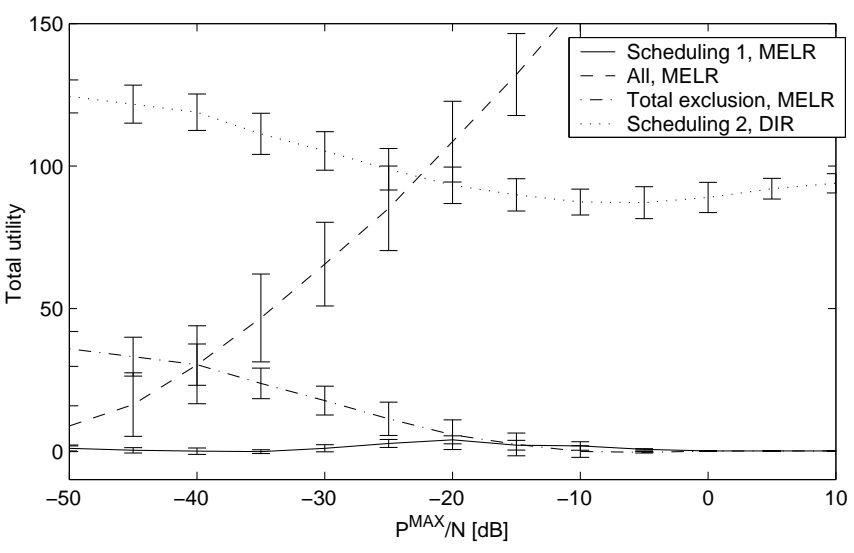

Fig. 12. Utilities of different routing and scheduling algorithms applied on random networks with non-uniform node distribution. We assume 40 nodes are uniformly distributed on the left half on the unit square, and 10 nodes on the right half. Source-destination pairs are uniformly chosen. On the $\mathrm{x}$ axis a relative power constraint $P^{M A X} / N$ is given. On the $y$ axis the differences between total utility of the reference approach (scheduling strategy 2 and the MELR routing) and the analyzed approach are given. 

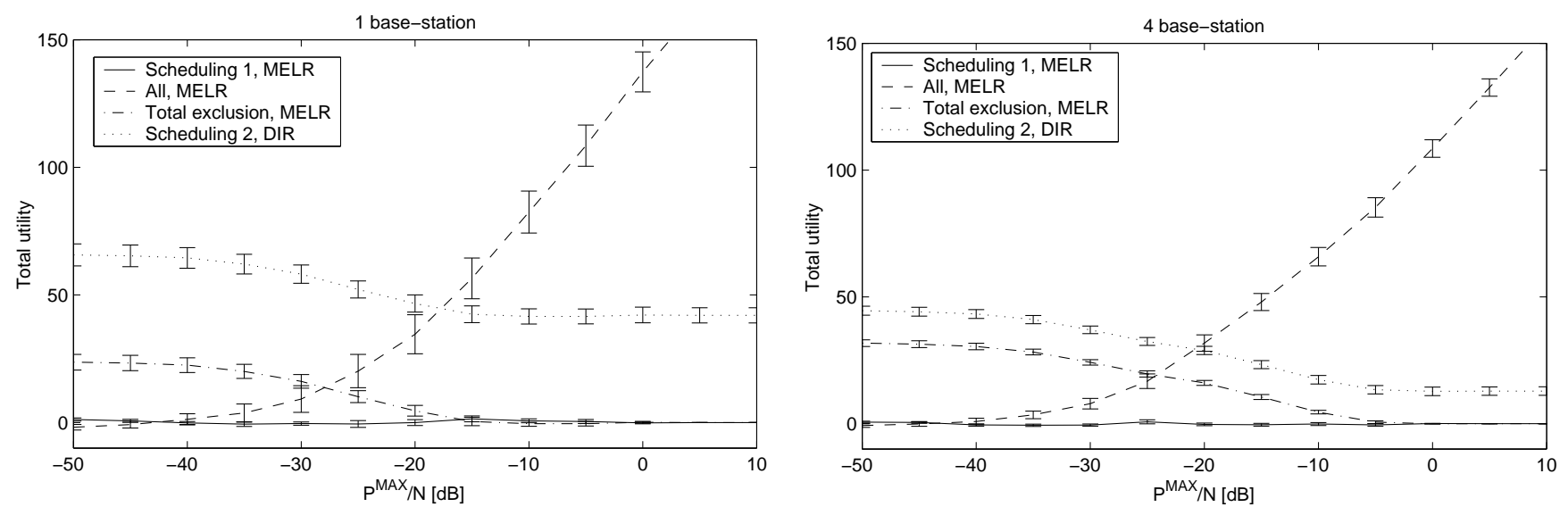

Fig. 13. Utilities of different routing and scheduling algorithms applied on homogeneous random networks with base-stations. We assume 50 randomly distributed nodes are sending data to the nearest of 1 (on the left) or 4 (on the right) base stations. On the $x$ axis a relative power constraint $P^{M A X} / N$ is given. On the y axis the difference between total utility of the reference approach (scheduling strategy 2 and the MELR routing) and the analyzed approach are given.
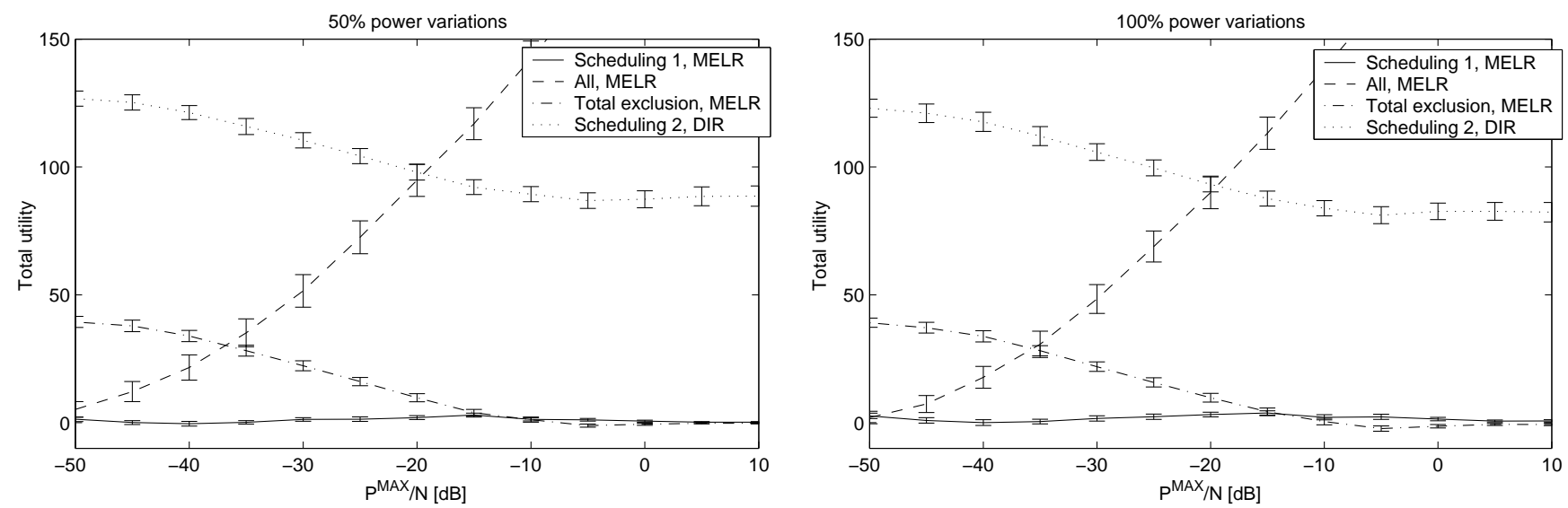

Fig. 14. Utilities of different routing and scheduling algorithms applied on homogeneous random networks with non-uniform maximal power constraints. We assume power constraints are uniformly distributed in the intervals of $\left[0.5 P^{M A X}, 1.5 P^{M A X}\right]$ (on the left) and $\left[0,2 P^{M A X}\right]$ (on the right). On the $\mathrm{x}$ axis a relative average power constraint $P^{M A X} / N$ is given. On the $y$ axis the differences between total utility of the reference approach and the analyzed approach are given. Here, scheduling strategy 2 and MELR routing are taken as the reference approach.

ing and routing strategies, and we search for the one that has the highest utility. We repeat the procedure for several random network topologies and for different values of power constraints. Examples of comparisons can be seen on Fig. 12 for non-homogeneous network topologies, Fig. 13 for nonhomogeneous traffic matrix, and Fig. 14 for non-uniform power constraints.

For all considered network scenarios we derive the same conclusions. We find that the optimal routing is the one with minimal hop length, which is MELR routing, regardless of the choice of the scheduling protocol. This confirms finding 4 . We also find that the optimal scheduling strategy is strategy 2 , regardless of the choice of the routing protocol. This confirms findings 1 and 2. Again, from the property of strategy 2 that the exclusion region depends only on the power constraint it follows that the optimal MAC protocol is independent of the routing strategy, which confirms finding 5 . We conclude that although our findings were initially derived for homogeneous networks, they are robust to changes in network characteristic and remain valid in non-homogeneous network scenarios.

Other interesting remarks can be made for the nonhomogeneous traffic case, depicted on Fig. 13. As we concluded, DIR routing is always worse than MELR, but it becomes more efficient as the number of base-stations increases, which is due to the fact that routes are are becoming shorter. Also, in the case of a network with non-uniform power constraints, depicted on Fig. 14, we find that, though MELR and MER routings do not give the same routes when power constraints are not uniform, in our tests both routings gave almost equal performance. 


\section{The Effects of Mobility and The Cost of Routing}

As discussed in the introduction, routing in the wireless networks might be expensive due to its cost, and to the mobility of nodes and instabilities of routes. In this section we investigate the impact of mobility on our findings. Again, we test all possible combinations of scheduling and routing strategies and find which one has the highest utility. We do the test on homogeneous and non-homogeneous network scenarios described above. We considered two levels of mobility, with packet loss probabilities of $10 \%$ and $25 \%$.

We obtain the same results in all analyzed cases. The routing with minimum hop length is the optimal one, regardless of the choice of scheduling. Scheduling strategy 2 is the optimal one for all routing strategies. As already discussed above, this confirms our findings.

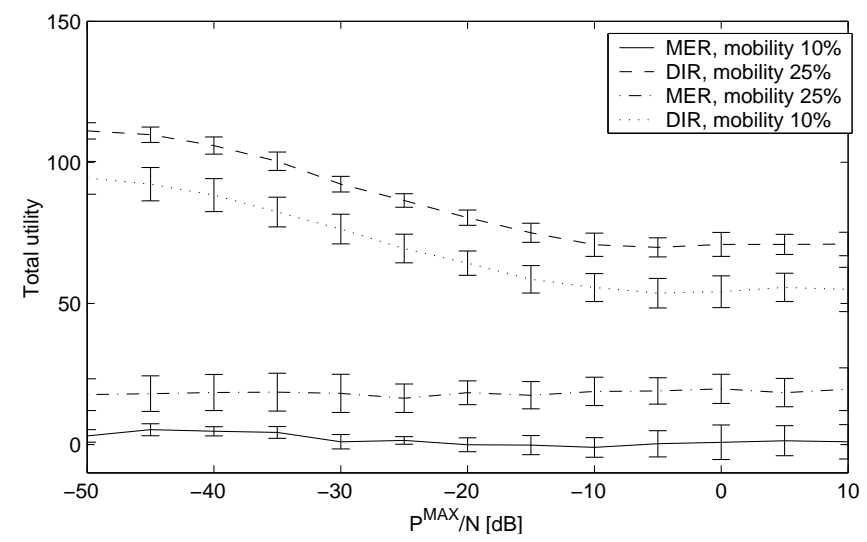

Fig. 15. Utilities of different routing algorithms applied on mobile homogeneous random networks and different maximal power constraints. Fraction of packets lost on each hop is $10 \%$ or $25 \%$. On the $x$ axis a relative power constraint $P^{M A X} / N$ is given. On the $y$ axis are the differences between total utility of the reference approach (MELR routing and scheduling strategy 2) and MER and DIR routing again with the optimal scheduling strategy 2 .

A snapshot of numerical results can be seen on Fig. 15, where we show utilities of different routings with the optimal scheduling, for different mobility levels. As noted above, MELR routing is the optimal one, and for a static case, it is equivalent to MER routing. As the mobility level increases, MER routing deteriorates, and DIR improves. We verified that for very high packet loss ratios due to mobility DIR will eventually become equivalent to MELR. However, this does not happen in realistic examples. Furthermore, as expected, mobility does not influence the optimal scheduling strategy as it does not change behavior in any other way but adding additional cost to the routes.

Another previously mentioned drawback of routing is its cost. We next discuss what the maximal cost of a routing is after which our findings do not hold any more. We say that a routing protocol consumes a constant fraction $C_{r}$ of rate of each link. It is obvious that it is not going to impact the optimal scheduling strategy, hence findings 1,2 and 5 will continue to hold. On the contrary, a sufficiently high value of this additional cost can penalize routing such that no routing (that is to say DIR) becomes the optimal strategy, thus invalidating finding 4. We are interested in finding for which values of $C_{r}$ this will happen.

According to (5) we have that the rate of a fbw $i$ is $f_{i}=$ $f_{i}^{\mathrm{nr}}\left(1-C_{r}\right)$, where $\mathbf{f}^{\mathrm{nr}}$ are the rates in the same network without routing costs. If a network consists of $I$ fbws, then the utility of the network with routing will be $U=I \log (1-$ $\left.C_{r}\right)+U^{\mathrm{nr}}$, or

$$
C_{r}=1-\exp \left(\left(U-U^{\mathrm{nr}}\right) / I\right)
$$

For example, for a network with 25 fbws, if the routing cost is $C_{r}=0.25$ then the decrease in utility comparing to the same network without routing cost is going to be around 7 . By inspecting the above presented graphs, and using (11), we estimate for which values of $C_{r}$ our finding 4 will cease to hold.

We first consider static networks. From Fig. 11 we see that the difference in utilities between DIR and MER (which is here equivalent to MELR) is between 70 and 90. From (11) we see that DIR becomes better when the cost of routing is $C_{r} \approx$ 0.85 . As $85 \%$ of the overhead can be considered very high for a routing protocol, we conclude that in realistic scenarios MELR is the optimal routing, and our finding 4 still holds. The same result holds for non-homogeneous networks.

Next, we consider mobile networks from Fig. 15, with a packet loss probability of $25 \%$. The difference in utilities between DIR and the optimal routing is more than 25. Again from (11) we conclude that DIR will become optimal for cost $C_{r}>0.63$. Both a packet loss ratio of $25 \%$ and a routing overhead of $63 \%$ still represent extreme values and we again conclude that, in realistic scenarios with mobility, our finding 4 still holds.

\section{COMPARISON TO NARROW-BAND NETWORKS}

An interesting question to discuss is if our findings for wideband networks can be applied to narrow-band networks. We give here a simple yet illustrative example why this is not possible.

We can derive a model of a narrow-band network similar to our model (5) of a wide-band network. In the narrow-band case, the rate of a link is not going to be a linear function of the SNR, but rather something like

$$
x_{l}^{n}=K \log \left(1+\mathbf{S N R}_{l}\left(\mathbf{p}^{n}\right)\right) .
$$

Let us consider the narrow-band model of a static ring. Using a similar techniques as in Section V, we can solve the optimization problem. For more details see [22].

On Fig. 16 on the left, the maximal rates achieved by different routings in an 18-node ring are depicted. We see that MER routing is better for small powers, and DIR routing is better for large powers. This is in contradiction to our finding 4 for UWB networks.

The numbers of active nodes in the optimal schedules for different routings are depicted on the right of Fig. 16. Although we verify that the optimal schedules for both MER and DIR routing do have the exclusion regions around each receiving node, we see from Fig. 16 that the sizes of these regions vary. The size of the exclusion region depends on the length of links, 

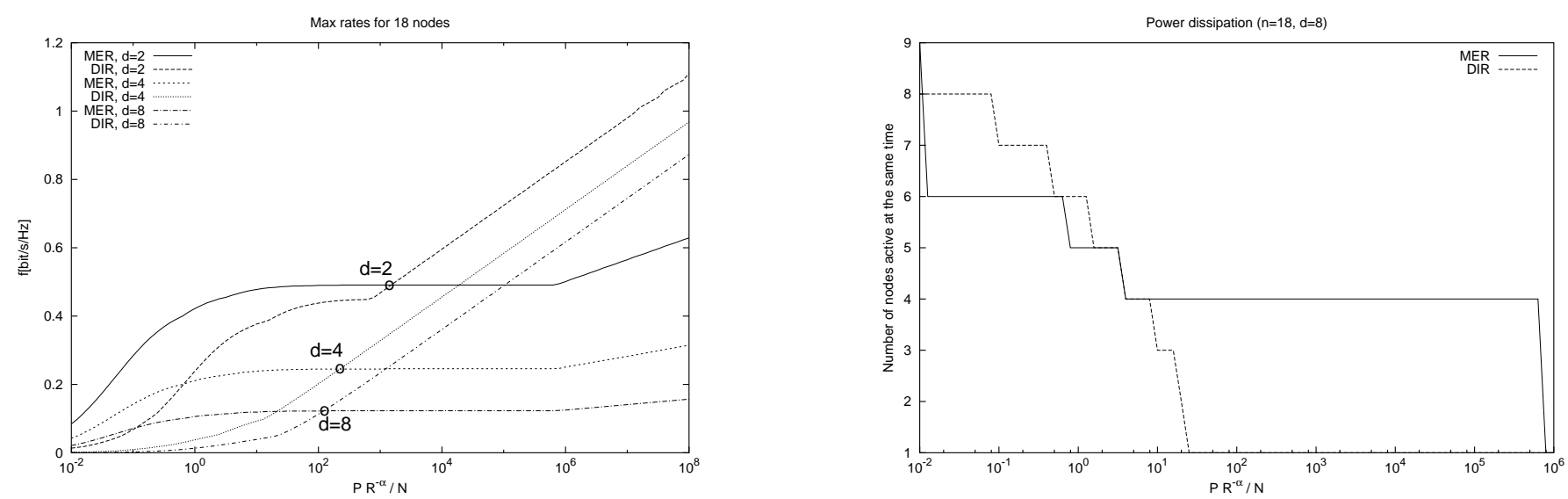

Fig. 16. On the left, maximum rates achieved by DIR and MER routing and different values of $d$ are depicted, for a ring of 18 nodes. In all cases, we consider the optimal schedule. We see that for small transmitting powers MER routing is better then DIR, and for large powers DIR becomes better. This is in contradiction with our finding 4 that MER is always the best. On the right we see the number of nodes active in one time slot. We see that it is not the same for different routing policies. This means that the sizes of exclusion regions depend on the routing policy and link lengths. This violates our findings 2 and 5.

and therefore depends on the choice of the routing protocol. This is in contrast to our findings 2 and 5 for UWB networks.

From the above results we see that in narrow-band networks, the optimal routing depends on the power constraints. The optimal scheduling depends on the length of links, and the choice of the routing. Thus, the design of the optimal narrowband networking architecture is essentially a non-separable joint optimization problem. This confirms the result in [30], which applies to 802.11.

On the contrary, our findings show that for wide-band networks the joint optimization problem can be solved separately for routing and MAC layers. This implies that the design of UWB networks is significantly simpler than for narrow-band.

\section{COnclusions And Future Work}

We have answered questions on what objective the MAC layer and the routing protocol should have in a multi-hop UWB ad-hoc network. We have presented a general model for joint scheduling, power allocation and routing optimization problem. We have derived the exact optimal power allocation for an arbitrary network, for any scheduling and routing strategy. We have analytically solved the scheduling and routing optimization problem for symmetric one-dimensional networks, and approximately by numerical simulations for arbitrary networks in a plane. Based on these solutions we have identified findings that we showed to hold quite generally in arbitrary UWB networks.

We first find that in a multi-hop UWB network, unlike in wide-band networks, the optimal MAC protocol does not depend on the choice of the routing. This finding emphasizes that a traditional layered network architecture is applicable to multi-hop wireless network, and that a MAC protocol can be designed regardless of the choice of the routing protocol.

We also find that for static networks, minimum energy route (MER) is the optimal route not only from the energy but also from the rate performance viewpoint. This finding holds even for routing protocol with high signaling overhead. When nodes are mobile, then the optimal route minimizes a function of dissipated energy and the number of hops on the route. Again, this finding holds for moderately high mobility and routing overheads.

Our last finding is about the optimal MAC design. We find that a node should maintain an exclusion region around it while receiving. All nodes in the exclusion region should remain silent during the reception, while nodes outside of this region may transmit in parallel. The size of this exclusion region depends only on the power constraints of the source of the transmission, and not on the length of the link or the positions of other nodes. Additionally, the receiver and its corresponding sender should adapt the rate of the communication according to the amount of noise and interference at the receiver. When a node is transmitting, it should do so with maximum power.

These findings give directions for implementations of routing and MAC protocol. A routing protocol should be based on a distributed shortest path algorithm considering inverse links' attenuations as the costs of links. To this end, any standard adhoc network routing protocol (AODV, DSR,...) could be used to calculate the shortest path to the destination. The cost of a link can be measured and updated during the transmission of every packet.

Our finding is that the optimal MAC protocol in an UWB network should be a combination of rate adaptation and mutal exclusion. The size of the exclusion region should be adapted to the parameters of a network. The fact that the optimal size of the exclusion region is defined solely on local information facilitates MAC protocol design. Details of the actual implementation of the protocols is for future work. Note that our findings suggest that there are fundamental reasons why re-using MAC protocols originally designed for narrowband (as envisioned by IEEE 802.15.3a) might not be a good 
idea. Another application lies in the area of low-power UWB networks (as proposed by 802.15.4a). Our results suggest that, for sufficiently low powers, the exclusion regions are going to be small enough that no exclusion protocol are necessary. Therefore, a simple all-at-once scheduling strategy without complex exclusion-region based signaling might be optimal for a class of low-power UWB networks [18]. Although our findings do not directly hold in narrow-band network, a similar analysis of a narrow-band network could be performed to improve the existing CSMA/CA protocols for narrowband networks (i.e. 802.11).

Although we have not directly discussed the issues of QoS in UWB networks in the sense of rate of power guarantees, our work gives important guidelines for design of QoS aware protocols. The maximization of rates in a QoS network is a fundamental concept, as well as fairness, which is typically implemented within a single QoS category. The actual integration of these concept in a QoS scheme is for future work.

\section{Different UWB Models}

As we mentioned in the introduction, there exist several UWB physical models, and we have primarily focused on the Win Scholtz model [33]. It would be interesting to discuss if our findings could be extended and applied to another emerging UWB model, the model of Souilmi, Knopp and Caire [25]. It differs in its coding scheme and transceiver architecture. A specificity is that it implements exclusion at the physical layer, thus physical layer signaling and scheduling cannot be separated as they are in our model. Hence our findings do not apply verbatim to their model.

However, there are indications that findings similar to ours might hold in [25]. Indeed, in [25], the authors have shown that the exclusion mechanism has to adapt to the number of nodes in the surrounding, but they have not analyzed it in further detail. Also, one may think that, since the behavior of an active link does not have to change due to a transmitting node that is very far away, the exclusion mechanism in [25] would in fact be needed only for nodes that are not too far apart. This resembles our findings about the exclusion regions and their optimal sizes. It remains for future work to refine our model in order analyze this generalization in detail.

\section{APPENDIX}

Lemma 1: For an arbitrary vector $\mu$ function $\sum_{i} \mu_{i} \mathbf{f}_{i}$ achieves maximum on set $\mathcal{F}$ and furthermore this maximum belongs also to $\mathcal{F}^{E}$.

Both function $\sum_{i} \mu_{i} \mathbf{f}_{i}$ and set $\mathcal{F}$ are convex, hence the maximum is attained in some $\mathbf{f}$. Since $\mathbf{f} \in \mathcal{F}$, we know there exist $\alpha=\left(\alpha^{1}, \cdots, \alpha^{N}\right), \mathbf{p}^{1}, \cdots, \mathbf{p}^{N}$ that satisfy (5).

We first analyze the case when $\mu \geq 0$, and we use the approach from [4], [5]. Without loss of generality, we fix all $\alpha, \mathbf{p}^{1}, \mathbf{p}^{2}, \cdots, \mathbf{p}^{N}$ except $p_{1}^{1}$, and we consider a function $p_{1}^{1} \stackrel{U}{\rightarrow}$ $\sum_{i} \mu_{i} \mathbf{f}_{i}$ as a function of a single free variable $p_{1}^{1}$. It is easy to verify that regardless of the values of other variables, $U\left(p_{1}^{1}\right)$ is always convex, hence the maximum is attained for $p_{1}^{1} \in$ $\left\{0, P^{M A X}\right\}$. Therefore, we have that $\mathbf{p}^{1}, \cdots, \mathbf{p}^{N} \in \mathcal{P}^{E}$, and $\mathbf{f} \in \mathcal{F}^{E}$.
Next we suppose, without loss of generality, that for some $m$ we have $\mu_{1}<0, \cdots, \mu_{m}<0$. Then clearly the optimal is to have $f_{1}=0, \cdots, f_{m}=0$ which is always feasible, regardless of the average rates of links $\overline{\mathbf{x}}$. Then by setting $\mu_{1}=$ $0, \cdots, \mu_{m}=0$, the new optimization problem has the same maximum as the old one, and we again have that $\mathbf{p}^{1}, \cdots, \mathbf{p}^{N} \in$ $\mathcal{P}^{E}$, and $\mathbf{f} \in \mathcal{F}^{E}$, which concludes the proof.

Proof of Theorem 1: We proceed by contradiction. Suppose there exists a point $\mathbf{f} \in \mathcal{F}$ that is not in $\mathcal{F}^{C}$. Then by the separating hyperplane theorem [32] there exists a hyperplane defined by $(\mathbf{c}, b)$ that separates $\mathbf{f}$ and $\mathcal{F}^{E}$, that is $\mathbf{c}^{T} \mathbf{f}>b$ and for all $\mathbf{g} \in \mathcal{F}^{E}, \mathbf{c}^{T} \mathbf{g}<b$. This on other hand means that f $\notin \mathcal{F}^{E}$ maximizes the utility function $U$ for $\mu=\mathbf{c}$, which contradicts with Lemma 1.

\section{REFERENCES}

[1] IEEE 802.15 WPAN High Rate Alternative PHY Task Group 3a. Multiband ofdm physical layer proposal. IEEE 802.15-03/267r5, July 2003.

[2] Pierre Baldi, Luca De Nardis, and Maria-Gabriella Di Benedetto. Modeling and optimization of uwb communication networks through a flexible cost function. IEEE Journal on Selected Areas in Communications, 20(9):1733-1744, December 2002.

[3] N. Bambos, S. Chen, and G. Pottie. Radio link admission algorithms for wireless networks with power control and active link quality protection. In Proceedings INFOCOM, 1995.

[4] R. Cruz and A.V. Santhanam. Optimal link scheduling and power control in cdma multihop wireless networks. In Globecom, 2002.

[5] F. Cuomo, C. Martello, A. Baiocchi, and F. Capriotti. Radio resource sharing for ad hoc networking with uwb. IEEE Journal on Selected Areas in Communications, 20(9):1722-1732, December 2002.

[6] G. Durisi and G. Romano. On the validity of gaussian approximation to characterize the multiuser capacity of UWB TH PPM. In IEEE Conference on Ultra Wideband Systems and Technologies, pages 151$161,2002$.

[7] T. Elbatt and A. Ephremides. Joint scheduling and power control for wireless ad-hoc networks. In Proceedings INFOCOM, 2002.

[8] P.K. Frenger, P. Orten, T. Ottosson, and A.B. Svensson. Rate-compatible convolutional codes for multirate DS-CDMA systems. IEEE Transactions on Communications, 47(6):828-836, June 1999.

[9] S.S. Ghassemzadeh and V. Tarokh. Uwb path loss characterization in residential environments. In IEEE Radio Frequency Integrated Circuits (RFIC) Symposium, pages 501-504, June 2003.

[10] A. Goldsmith and S.B. Wicker. Design challenges for energy-constrained ad hoc wireless networks. IEEE Wireless Communications, 9(4):8-27, August 2002.

[11] P. Gupta and P.R. Kumar. The capacity of wireless networks. IEEE Transactions on Information Theory, 46(2):388-404, March 2000.

[12] J. Hagenauer. Rate-compatible punctured convolutional codes (RCPC codes) and their applications. IEEE Transactions on Communications, 36(4):389-400, April 1988

[13] X. Huang and B. Bensaou. On max-min fairness and scheduling in wireless ad-hoc networks: Analytical framework and implementation. In Proceedings MobiHoc'01, Long Beach, California, October 2001.

[14] D.B. Johnson and D.A. Maltz. Dynamic source routing in ad hocwireless networks. In T. Imielinski and H. Korth, editors, Mobile Computing, chapter 5, pages 153-181. Kluwer Publishing Company, 1996.

[15] Julian, Chiang, O'Neill, and Boyd. Qos and fairness constrained convex optimization of resource allocation for wireless cellular and ad hoc network. In Proceedings INFOCOM, 2002.

[16] V. Kawadia, S. Narayanaswamy, R. Rozovsky, R.S. Sreenivas, and P.R. Kumar. Protocols for media access control and power control in wireless networks. In Proceedings of the 40th IEEE Conference on Decision and Control, pages 1935-1940, Orlando, FL, Dec. 4-7 2001.

[17] F.P. Kelly, A.K. Maulloo, and D.K.H. Tan. Rate control in communication networks: shadow prices, proportional fairness and stability. Journal of the Operational Research Society, 49:237-252, 1998.

[18] J. Y. Le Boudec, R. Merz, B. Radunovic, and J. Widmer. A mac protocol for uwb very low power mobile ad-hoc networks based on dynamic channel coding with interference mitigation. Technical Report IC/2004/02, EPFL-DI-ICA, January 2004. 
[19] Related Matlab scripts, http://icapeople.epfl.ch/bradunov/jsac.zip.

[20] T. et al. Nandagopal. Achieving mac layer fairness in wireless packet networks. In Proceedings MobiCom'00, Boston, Massachusetts, August 2000.

[21] C.E. Perkins and E.M. Royer. Ad-hoc on-demand distance vector routing. In In Proceedings of IEEE WMCSA'99, pages 90-100, New Orleans, LA, February 1999.

[22] B. Radunović and J.-Y. Le Boudec. Joint scheduling power control and routing in symmetric one dimensional multi hop wireless networks. In WiOpt'03: Modeling and Optimization in Mobile, Ad Hoc and Wireless Networks, INRIA Sophia-Antipolis, France, March 3-5 2003.

[23] B. Radunović and J.-Y. Le Boudec. Rate performance objectives of multi-hop wireless networks. In Proceedings INFOCOM, 2004.

[24] A. Rajeswaran and R. Negi. Capacity of power constrained ad-hoc networks. In Proceedings INFOCOM, 2004.

[25] Y. Souilmi, R. Knopp, and G. Caire. Coding strategies for uwb interference- limited peer-to-peer networks. In WiOpt'03: Modeling and Optimization in Mobile, Ad Hoc and Wireless Networks, INRIA SophiaAntipolis, France, March 3-5 2003.

[26] L. Tassiulas and A. Ephremides. Jointly optimal routing and scheduling in packet radio networks. IEEE Transactions on Information Theory, 38(1):165-168, January 1992.

[27] L. Tassiulas and S. Sarkar. Max-min fair scheduling in wireless networks. In Proceedings INFOCOM, 2002.

[28] I.E. Telatar and D.N.C. Tse. Capacity and mutual information of wideband multipath fading channels. IEEE Transactions on Information Theory, 46(4):1384-1400, 2000.

[29] S. Toumpis and A.J. Goldsmith. Capacity regions for wireless ad hoc networks. IEEE Transactions on Wireless Communications, 2(4):736748, July 2003.

[30] S. Toumpis and A.J. Goldsmith. Performance, optimization, and crosslayer design of media access protocols for wireless ad hoc networks. In International Conference on Communications (ICC), pages 2234-2240, Anchorage, AK, May 2003.

[31] S. Verdu. Spectral effi ciency in the wideband regime. IEEE Transactions on Information Theory, 48(6):1319-1343, 2002.

[32] R. Webster. Convexity. Oxford University Press, 1994.

[33] M. Win and R. Scholtz. Ultra-wide bandwidth time-hopping spreadspectrum impulse radio for wireless multiple-access communications. IEEE Transactions on Communications, 48(4):679-691, April 2000. 